EGG-CODP -10230

DE92 018045

\title{
Vectum User's Guide
}

Fred R. McClurg

Vincent A. Mousseau

April 1992

\section{Idaho National Engineering Laboratory EG\&G Idaho, Inc. Idaho Falls, Idaho 83415}

Prepared for the

U.S. Department of Energy

Field Office, ldaho

Under DOE Contract No. DE-AC07-761D01570 


\section{Abstract}

This user's guide is the documentation for vectum: the easy to use, flexible, multi-purpose, 2D velocity vector plotting package based on the NCAR Graphic: libraries. 
Vectum User's (juide

\section{Acknowledgments}

The authors would like to express their appreciation to the following:

Technical Services, Visualization and Imaging Team

Computational Fluid Dynamics Unit

Partial Support from the Long-term Research Initiative in Computational Mechanics 
Vectum User's Guide

2.1 .21 Theta parameter . . . . . . . . . . . . . . . . 17

2.1 .22 Scarro parancter . . . . . . . . . . . . . . . . . . 18

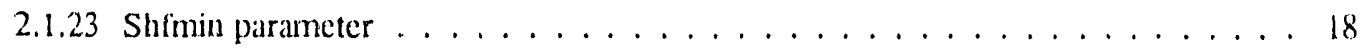

2.1 .24 Altchr parameter . . . . . . . . . . . . . . . . . 18

2.1 .25 Arwchr parameter . . . . . . . . . . . . . . . . 22

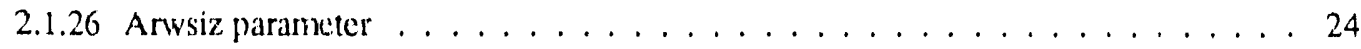

2.1 .27 Arwang paraneter . . . . . . . . . . . . . . . . . . 24

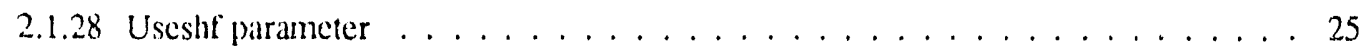

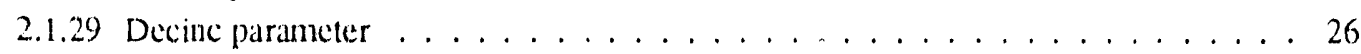

2.1 .30 vecrnin parameter . . . . . . . . . . . . . . . . . . . . . 29

2.1 .31 Optmin parameter . . . . . . . . . . . . . . . . . . . . . . . . . . 29

2.1 .32 vecmax paraneter ........................ 30

2.1 .33 optnax parameter . . . . . . . . . . . . . . . . . . . . .

2.1 .34 Movie parameter. . . . . . . . . . . . . . . . 33

2.2 Geometry latabase . . . . . . . . . . . . . . . . . . . . . . 33

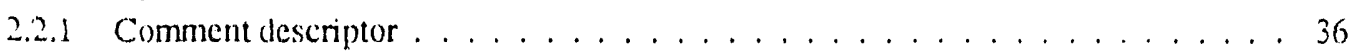

2.2 .2 Dash descriptor . . . . . . . . . . . . . . . . . 36

2.2 .3 Line descriptor . . . . . . . . . . . . . . . . . . . . . . . . . . . . 37

2.2 .4 String descriptor $\ldots \ldots \ldots \ldots \ldots \ldots$

2.2 .5 Arc (circle) descriptor . . . . . . . . . . . . . . . . . . . 4()

2.2 .6 Curve $($ spline descriptor . . . . . . . . . . . . . . . . . . 41

2.3 vector latabase . . . . . . . . . . . . . . . . . . . . . . 42

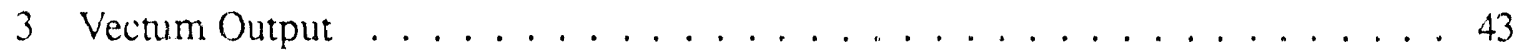

3.1 Displaying to a monitor . . . . . . . . . . . . . . . . . . 43

$3.1 .1 \times 11$ driver . . . . . . . . . . . . . . . . . 43

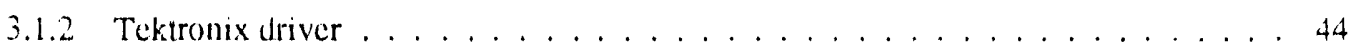

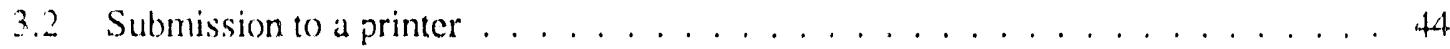

3.2 .1 PostScript driver . . . . . . . . . . . . . . . . . . . . . . . . 44

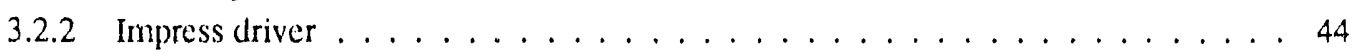

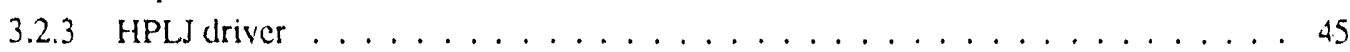

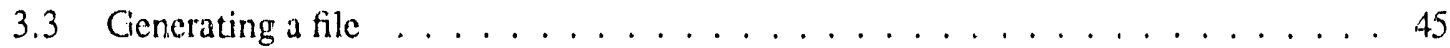

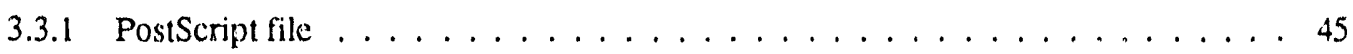

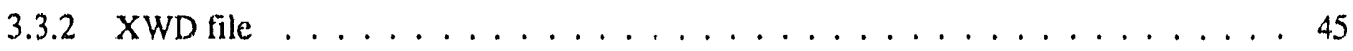

3.3 .3 Sun Raster file . . . . . . . . . . . . . . . . . . 46

3.3 .4 Encapsulated PostScript file . . . . . . . . . . . . . . . . . . . 46

References . . . . . . . . . . . . . . . . . . . . . . 49

Glossary . . . . . . . . . . . . . . . . . . 51 


\section{Table of Contents}

Abstract . . . . . . . . . . . . . . . . . iii

Acknowledgments ...................... . iv

Table of Contents . . . . . . . . . . . . . . . . . . v

List of Figures . . . . . . . . . . . . . . . . . . . . . vii

Vectum User's Guide . . . . . . . . . . . . . . . . . . . . . 1

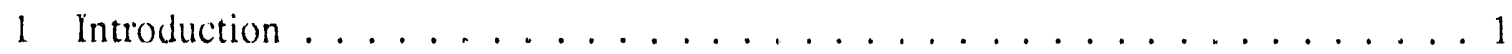

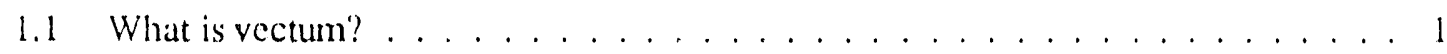

1.1.1 What are the distinguishing features? . . . . . . . . . . . 1

1.1 .2 How does it work? . . . . . . . . . . . . . . . . . . 1

1.2 Is vectum available? . . . . . . . . . . . . . . . . . . I

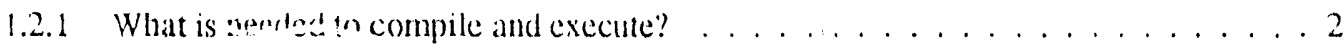

1.2 .2 How can I get a copy? . . . . . . . . . . . . . . . . 2

1.2 .3 How do 1 compile the source? . . . . . . . . . . . . . . . . . 3

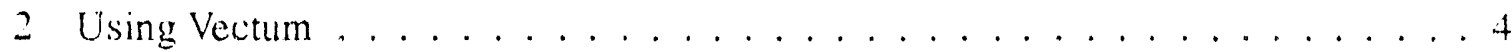

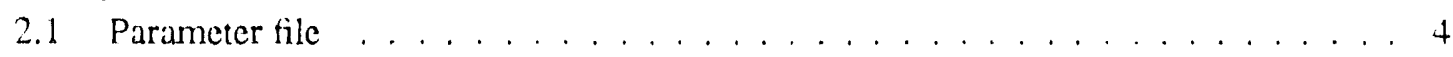

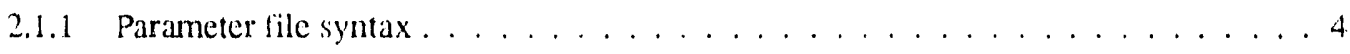

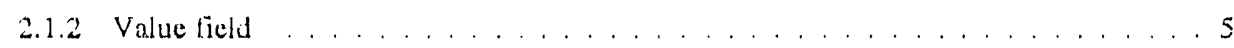

2.1 .3 Comment field ....................... 5

2.1 .4 Title parameter . . . . . . . . . . . . . . . . . 5

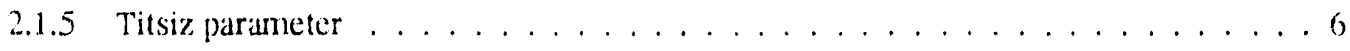

2.1 .6 Filein parameter . . . . . . . . . . . . . . . . 6

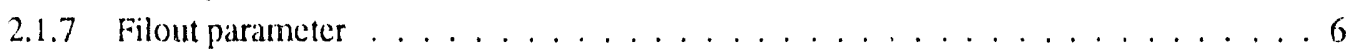

2.1 .8 Filgeo parameter ...................... 6

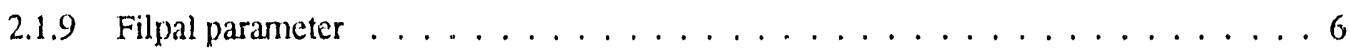

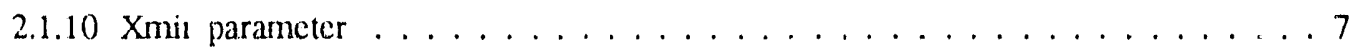

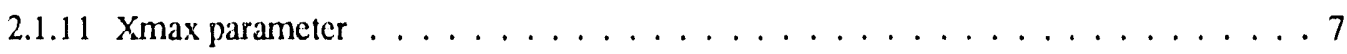

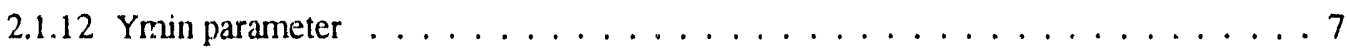

$2.1 .13 Y \max$ parameter $\ldots \ldots \ldots \ldots \ldots \ldots \ldots$

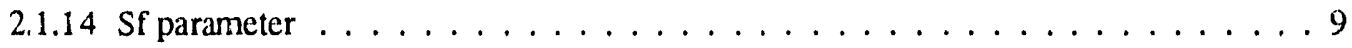

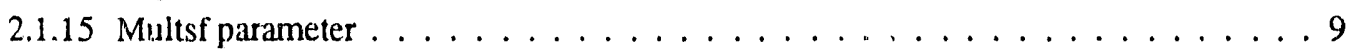

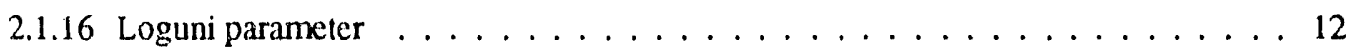

2.1 .17 Frac parameter . . . . . . . . . . . . . . . . . . 14

2.1 .18 Flex paranteter . . . . . . . . . . . . . . . . . . . 15

2.1 .19 Useaxs parameter . . . . . . . . . . . . . . . . 16

2.1 .20 Useleg parameter . . . . . . . . . . . . . . . . . . . 17 


\section{List of Figures}

FIGURE 1, Components of a viewport window $\ldots \ldots \ldots \ldots \ldots$

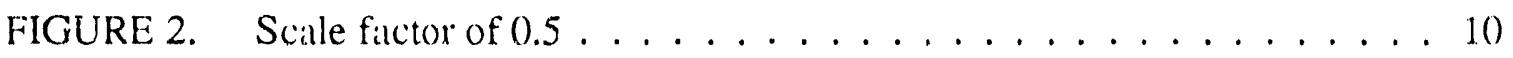

FIGURE 3. Scale fictor of $2.0 \ldots \ldots \ldots \ldots \ldots \ldots$

FIGURE 4. Unit vector shatt scaling $\ldots \ldots \ldots \ldots \ldots \ldots \ldots$

FIGURE 5. Normal vector shaft scaling . . . . . . . . . . . . . 13

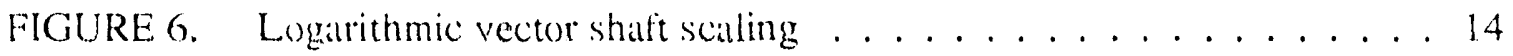

FIGURE 7. Equal unit scaling in the $\mathrm{x}$ and $\mathrm{y}$ directions . . . . . . . 15

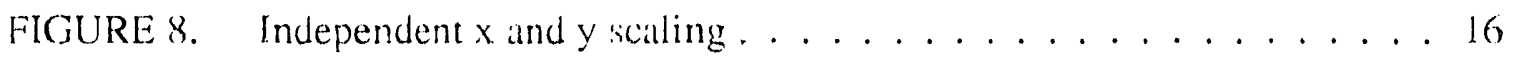

FIGURE 9. Line arrowhead parameters . . . . . . . . . . . . 17

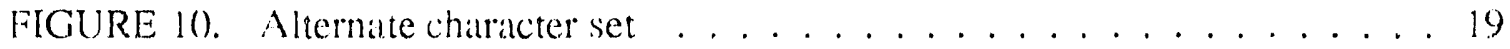

FIGURE 11. Alternate character set (cont.) . . . . . . . . . . . . . . . 20

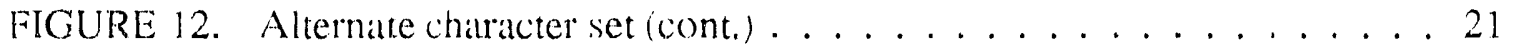

FIGURE 13. Alternate character set (cont.) . . . . . . . . . . . . . . 22

FIGURE 14. Characters in the same function code family . . . . . . . . 23

FIGURE 15. Characters in differing function code families . . . . . . . . . 24

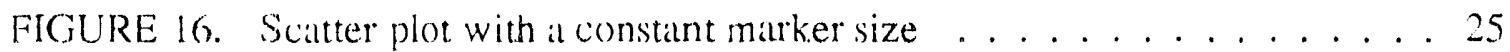

FIGURE 17. Sciatter plot with a varriable marker size . . . . . . . . . . 26

FIGURE 18. Line arrowheads with decimation factor of $1 \ldots \ldots$. . . . . . 27

FIGURE 19. Line arrowheads with decimation factor of $5 \ldots \ldots \ldots 28$

FIGURE 20. Specifying a range using a logical operator . . . . . . . . . . . 29

FIGURE 21 . Finite range specified $\ldots \ldots \ldots \ldots \ldots \ldots \ldots$

FIGURE 22. Minimum range specified $\ldots \ldots \ldots \ldots \ldots \ldots \ldots \ldots$

FIGURE 23. Maximum range specified $\ldots \ldots \ldots \ldots \ldots \ldots \ldots \ldots$

FIGURE 24. Infinite range specified $\ldots \ldots \ldots \ldots \ldots \ldots \ldots$

FIGURE 25. Miscellaneous geometry defined by the geometry database $\ldots \ldots 35$

FIGURE 26. Geometry drawn using the dash descriptor . . . . . . . . . . . 37 
Vectum User's Guide

FIGURE 27. Triangular grid mesh drawn using the line descriptor . . . . . . . . 38

FIGURE 28. Geometry drawn using the string descriptor . . . . . . . . . . . 39

FIGURE 29. Geometry drawn using the arc (circle) descriptor . . . . . . . . . . 40

FIGURE 30. Geometry drawn using the curve (spline) descriptor . . . . . . . . . . 41

FIGURE 31. Coordinates of a velocity vector . . . . . . . . . . . . 42

FIGURE 32. Imported Sun Raster file . . . . . . . . . . . . . . . 46

FIGURE 33. Imported Encapsulated PostScript file . . . . . . . . . . . . 47 


\section{Vectum User's Guide}

\section{Introduction}

This document is intended to serve as a general reference for vectum, the vector utility.

\subsection{What is vectum?}

Vectum is an easy to use. flexible, multi-purpose, 2D velocity vector, plotting packinge. It is also capable of generating particle plots (scatter plots), xy plots, and geometries.

\subsubsection{What are the distinguishing features?}

The program vectum allows the user to display irregular vector fields quickly and ealsily. It also gives the user the flexibility of modifying the characteristics of the vectors without recompilation.

Vectum uses the NCAR Graphics Libraries to generate publication quality output of a $2 D$ vector field. All of the NCAR Graphics utilities can be used with the output from vectum.

\subsubsection{How does it work:?}

Vectum uses a parameter tile to set the program 's behavior. This file controls the scalling of the vectors, annotation, dataset filename, and several other details. After determining the filename specified in the parameter file, vectum reads the ASCII database of irregular vector values and gene ates a NCAR CGM (Computer Graphics Metafile) file that can be displayed and plotted using the NCAR Graphics utilities.

\subsection{Is vectum available?}

The source for vectum is freely available to Idaho National Engineering Laboratory (INEL.) employees. 
Vectum Usicr's Ciuide

\subsubsection{What is needed to compile and execute?}

A FORTRAN 77 (f77) compiler and the NCAR Graphics Libraries and utilities.

\subsubsection{How can I get a copy?}

A compressed tar file of the source code is available through anonymous ftp on jade (134.2().8.49). The following work session outlines the process of getting a copy of that compressed tar file.

Make sure that your present working directory (pwd) is where you want the source located:

$$
\begin{aligned}
& \text { anmals\% mkdix sxc } \\
& \text { anllast od src }
\end{aligned}
$$

Transfer the file to your workstation:

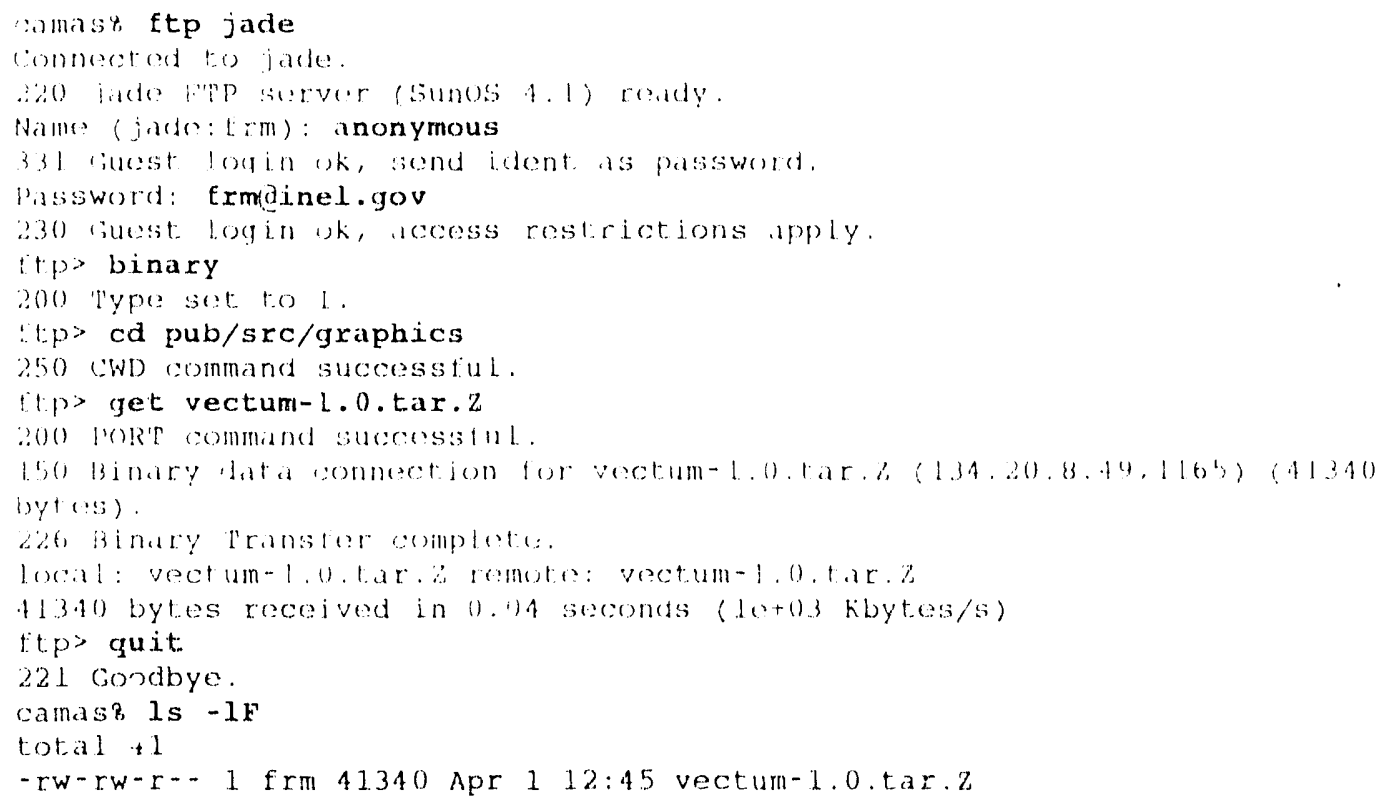

Uncompress and extract the source from the tar file:

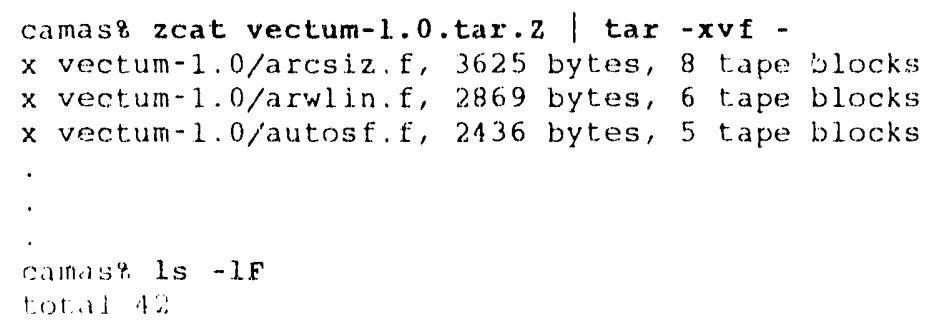




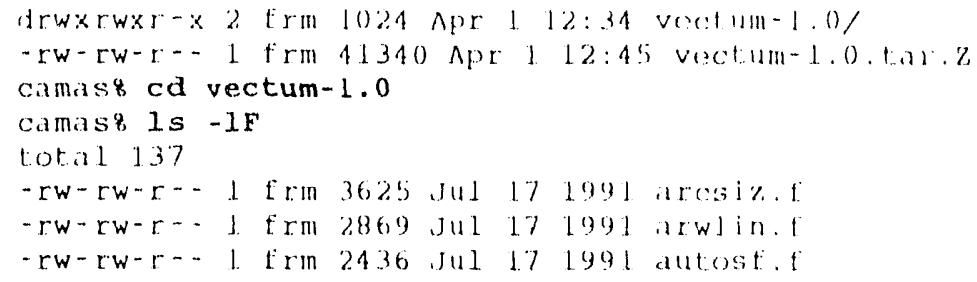

\subsubsection{How do I compile the source?}

After extracting the source from the compressed tal file, you will need to generate the program by compiling the source. A makefile is included for convenient compilation.

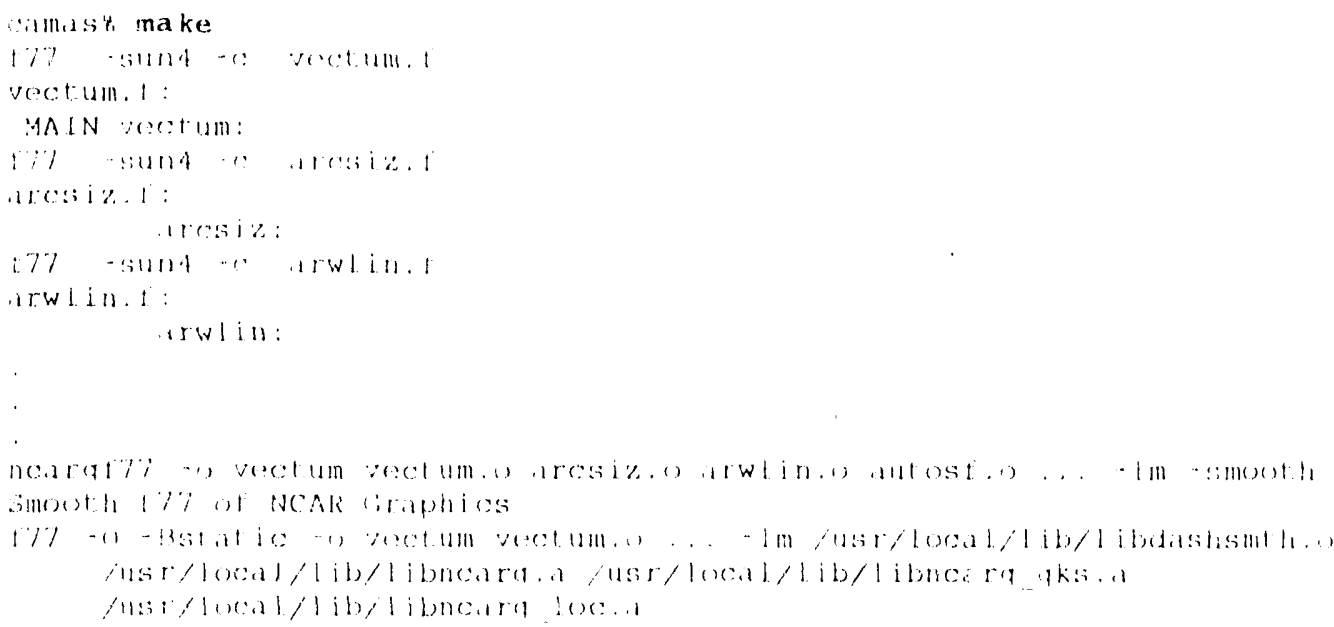

Upon successful compilation, the executable vectum should be generated.

camas' is $-1 F$ vectum

$-r w \times r w x-x 1$ trm 573440 Apr 1 12:53 vectum*

After generating the binary, the object files are no longer needed:

camast $\operatorname{rrn} * 0$

At this point you may wish to contact your system administrator to have the program installed in a location that everyone on your machine (or network) can access. 


\section{Using Vectum}

The following is a description for using the velocity vector package, vectum.

\subsection{Parameter file}

Upon execution, vectum reads an ASCII parameter file named "default.vec" in the user"s present working directory (pwd). The parameter file contains information that the user can modify to control how the program is executed and how the resulting vector field is displayed. The following is a listing of an example parameter file:

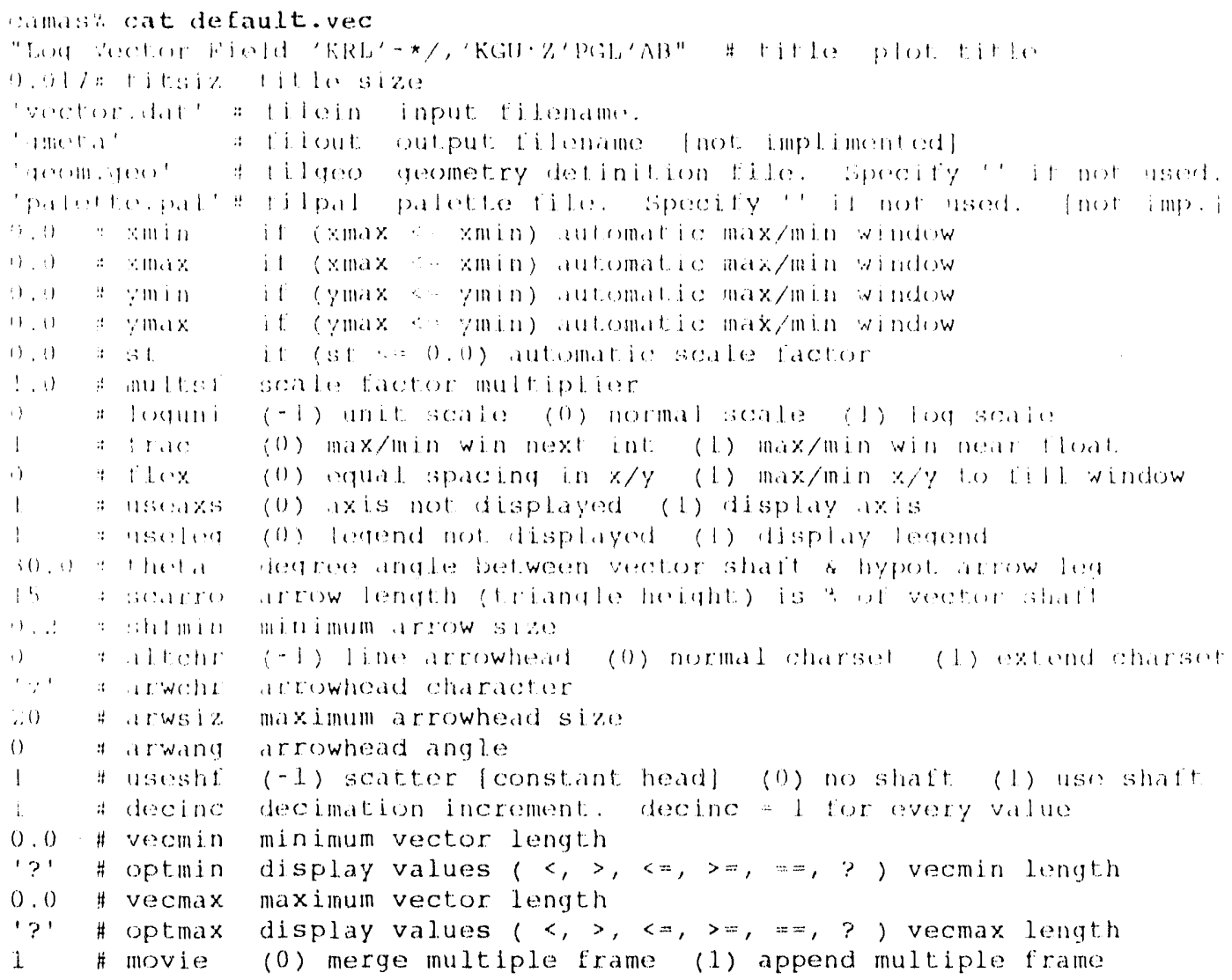

\subsubsection{Parameter file syntax}

Each line in the parameter file controls a different aspect of the program. Each line is necessary and the order of the lines must not be changed. The syntax of the parameter file is as 
follows:

$$
\text { value \# variable (0) option one (1) option two ... }
$$

As far as the program is concerned, there are really only two fields to each line. The value field and the comment field. The fields are delinited by spaces or tabs.

\subsubsection{Value field}

The value is the furst field on the line and can contain an integer, floating point, or chatracter type variable depending upon the context. The value field must transcribe to the following rules:

C) Fields using an integer context, should not contain points or characters.

) Fields using a floating point context, should contain one point and ao charalcters (except the exponential informations.

i) Fields using a character string context, should begin in column ont and be surrounded with single quotes 1 ') or double yuotes:").

\subsubsection{Comment field}

The comment is the second field on the line and begins with the symbol if. Everything following the symbol \# is ignored by vectum. The comment field contans several pieces of information that is descriptive of the value tield.

The variable rag is the first word in the comment field. It is used as a cryptic handle ti) describe the purpose of the value tield. In this documentation, each parameter will be bisted according to it's variable tag.

The option tag follows the variable tag in the comment field It is used to inform individuals regarding the setting of the value field.

\subsubsection{Title parameter}

The title parameter is the string that is centered at the top of the plot. The title parameter is of type character string. Note the following example:

"Vector Field 'KRL'-*/,'KGU'Z'PGL'AB" \# title plot tit.le

The title string must be enclosed with single quotes ( ') or double quotes ("). Any character can be used within the string except a single yuote. double yuote or grave (' I symbol. The grave symbol is reserved to display special chataters. See Section 2.1.25, un page ..2 for 
Vecium Uscris inide

al description of the Alternate Character Set.

\subsubsection{Titsiz parameter}

The titsiz parameter controls the size of titie string centered at the top of the plot. The titsiz parameter is of type float. Note the following example:

$$
0.017 \text { \# titsiz title size }
$$

\subsubsection{Filein parameter}

The filein parameter is the filename of the vector database. See Section 2.3, on page 42 for the tile format st the vector database. The filein paraneter is of type character string. Note the tollowing example:

$$
\text { 'rectordat' \# tilein int tilename. }
$$

\subsubsection{Filout parameter}

The filout parameter is the filename of the CGM file output by vectum. The filout parameter is of type character string. Note the following example:

$$
\text { 'gmeta' \# filout output tilename [not impjemented] }
$$

This parameter is not currently implemented programatically within vectum. The defiult ouput COM tilename "gmeta" can be changed to a user specified name by setting the following environment varible:

$$
\text { host setenv NCARG_GKS_oUTPUT mytile.gom }
$$

\subsubsection{Filgeo parameter}

The filgeo parameter is the name of the geometry database. See Section 2.2, on page 33 for a the file format of the geometry database. The filgeo parameter is of type character string. Note the following example:

$$
\text { 'geom.geo' \# filgeo geom file specify' ' if not used. }
$$

\subsubsection{Filpal parameter}

The filpal parameter is the name of the palette database. The filpal parameter is of type character string. Note the following example: 
'palette.pal' \# filpal palette Specify' ' if not user.

This parameter is not currently implemented.

\subsubsection{Xmin parameter}

The ${ }^{2}$ r in parameter is the minimum value of the viewport window in the $x$ direction. The . $m$ min parameter is of type float. See Figure 1, on page 8 for an illustration of $x m$ in orn a viewport window. Note the following example:
0.0
\# $x \mathrm{~min}$
if: $(x \max <=x \min )$
auto max/min window

If the value of the vinax parameter is less than or equal to the imin parameter, vectum will automatically maximize/minimize the viewport so that the entire data field will fill the window.

\subsubsection{Xmax parameter}

The imax parameter is the maximum value of the viewport window in the $\mathrm{x}$ direction. The xmax parameter is of type float. See Figure 1, on page 8 for an illustration of amax on a viewport window. Note the following example:

$$
\text { o.j \# zmax if (xmax } s=x \text { min) alito } \max / \text { injin window }
$$

If the value of the imax parameter is less than or equal to the umin parameter, vectum will automatically maximize/minimize the viewport so that the entire datal field will fill the window.

\subsubsection{Ymin parameter}

The ymin parameter is the minimum value of the viewport window in the $y$ direction. $T$ l e $y$ min parameter is of type float. See Figure 1 , on page 8 for an illustration of ymin on a view.port window. Note the following example:

$$
\text { o.c \# ymin if (ymax }<=y \min \text { ) auto } \mathrm{max} / \mathrm{min} \text { window }
$$

If the value of the ymax parameter is less than or equal to the ymin parameter, vectum will automatically maximize/minimize the viewport so that the entire data field will fill the window. 
Vectum User's (juide

\subsubsection{Ymax parameter}

The ymax parameter is the maximum value of the viewport window in the $y$ direction. The ymax parimeter is of type float. See Figure 1, on page 8 for an illustration of ymax on a viewport window. Note the following example:
0.0
\# ymax
i.f ( $y \max <=y \min$ ) auto $\max / \mathrm{min}$ window

If the value of the ymax parameter is less than or equal to the ymin parameter, vectum will automatically maximize/minimize the viewport so that the entire data field will fill the window.

FIGURE 1. Components of a viewport window

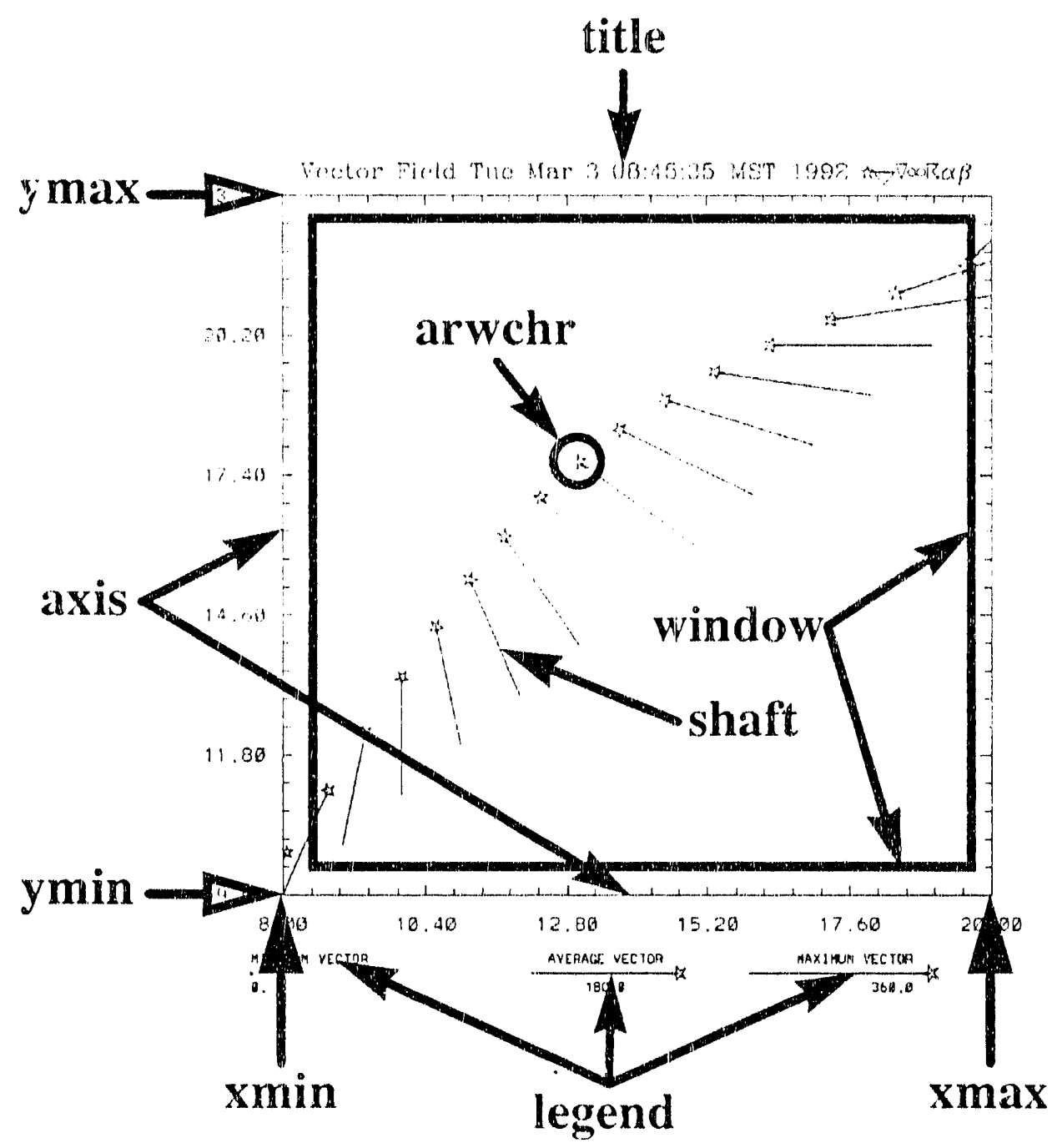




\subsubsection{Sf parameter}

The $s f$ parameter is the scale factor vectum uses inter:ally to make the vectors a reasonable size. The $s f$ parameter is of type float. Note the following example:

$$
0.0 \text { sf if (sf } s=0.0 \text { ) automatic scale factor }
$$

If the value of the $s f$ parameter is less than or equal to zero, vectum will automatically determine the scale factor and report the calculated value to the standard output. You may want to use this parameter to maintatin a common scale factor between two piots that are to be merged together. You may also want to use this parameter to eliminate the automatic vector shatt scaling in order to compare two different datasets. Figures 18 and 19 on pages 27 and 28 were generated by setting the sf parameter on the second plot (Figure 19) to the value that was calculated and reported to standard out by the first plot (Figure 18).

\subsubsection{Multsf parameter}

The multsf parameter is the scale factor multiplier: This parameter enables the user to easily scale the size of the vectors. The multsf parameter is of type Hoat. Note the following example:
1. 0
\# multsf
scale Eactor multiplier 
Yectum User's Guide

If the value of the multsf parameter is less than one, vectum will reduce the length of the vector shaft. See Figure 2.

FIGURE 2. Scale factor of 0.5

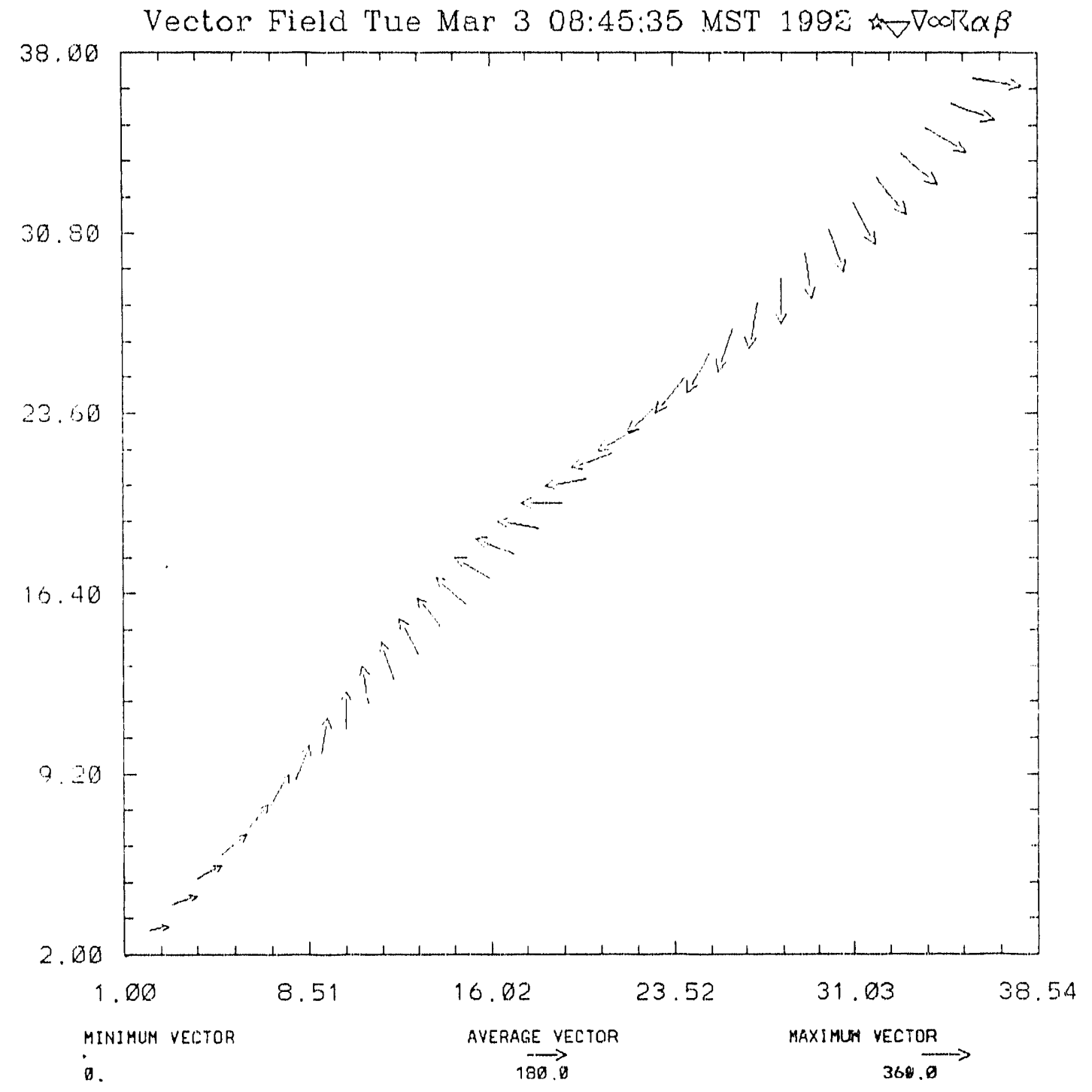


If the value of the multsf parameter is greater than one, vectum will enlarge the length of the vector shaft. See Figure 3.

FIGURE 3 . Scale factor of $\mathbf{2 . 0}$

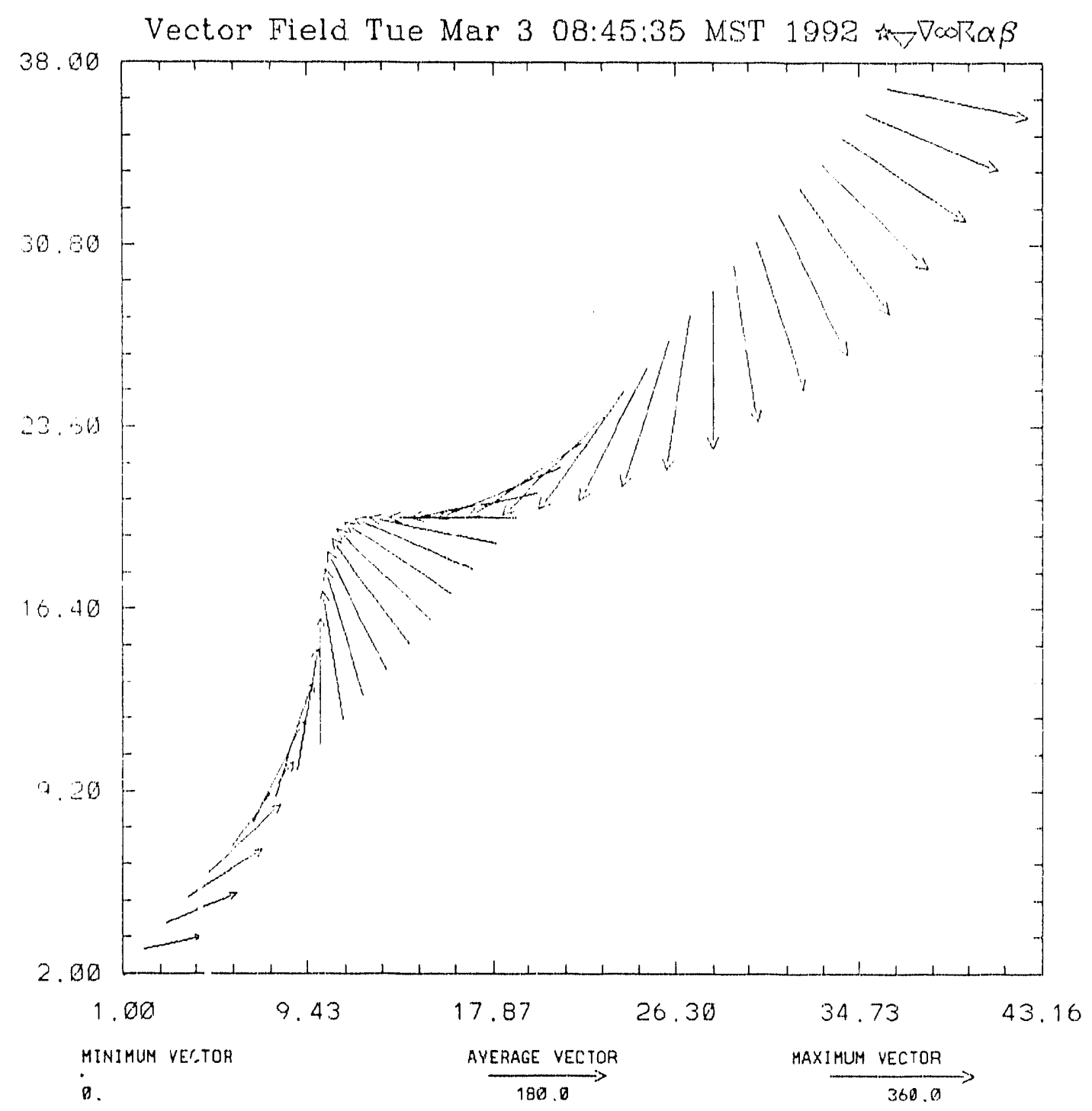

The multsf parameter should never be zero $(0.0)$ or less than zero. 


\subsubsection{Loguni parameter}

The loguni parameter is a three way switch for displaying unit vectors, normal scaled vectors, and log scaled vectors. The loguni parameter is of type integer. Note the following eximple:

$$
0 \text { \# loguni (-1) unit vector (0) normal (1) log }
$$

If the value of the loguni parameter is negative one $(-1)$, vectum will set the vector shaft scaling to unit scaling (see Figure 4). Unit scaled vectors have a constant vector length and indicate only the vector direction (and not the intensity). Zero length vectors will not be displayed due to the age old question, "What direction do zero length vectors go?".

\section{EICURE 4. Unit vector shaft scaling}

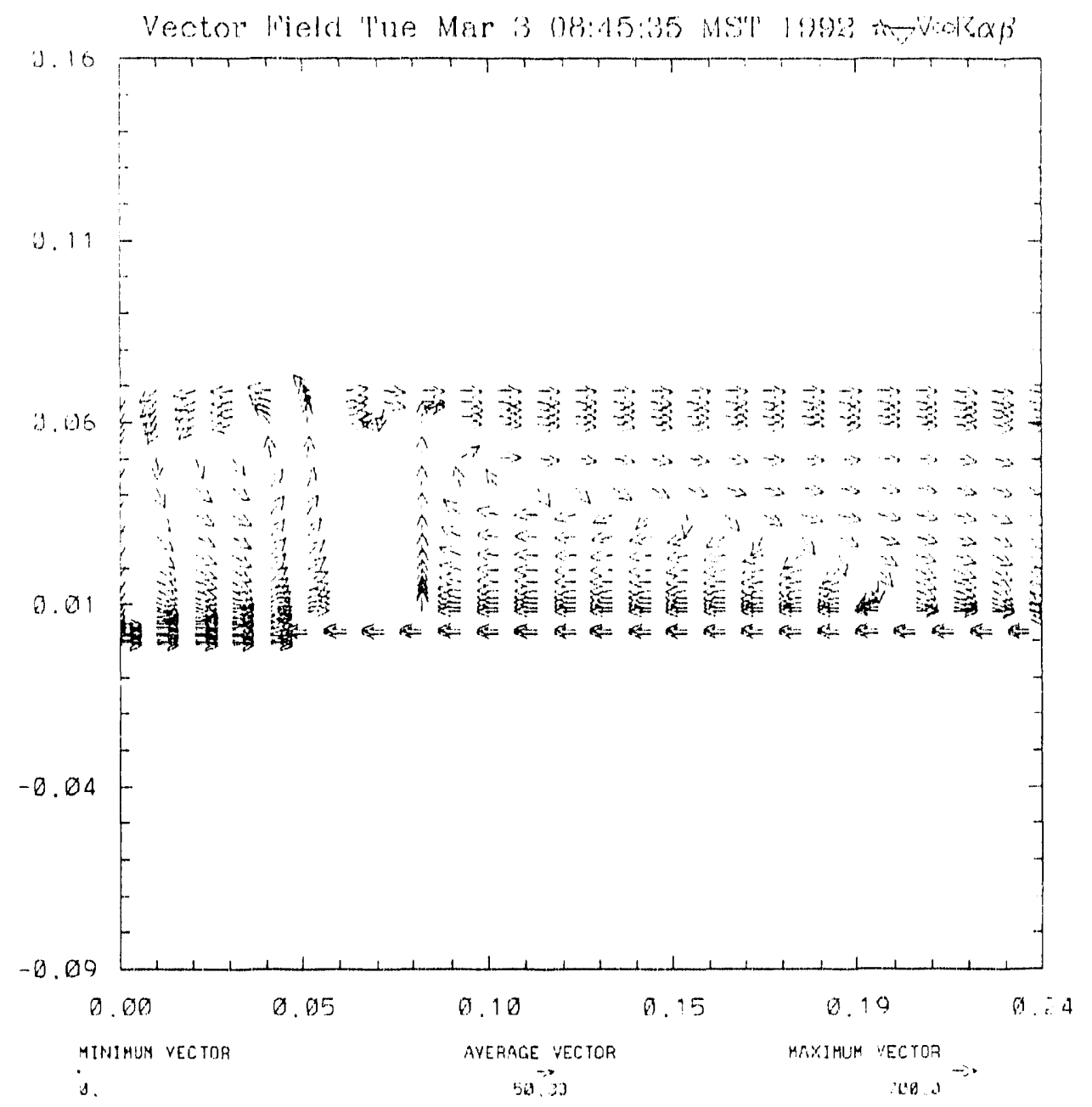


If the value of the logumi parameter is zero (0), vectum will set the vector shaft scaling in proportion to the $u / v$ values. See Figure 5.

FIGURE 5. Normal vector shaft scaling

Vector Field Tue Mar 3 08:45:35 MST 1992 avor

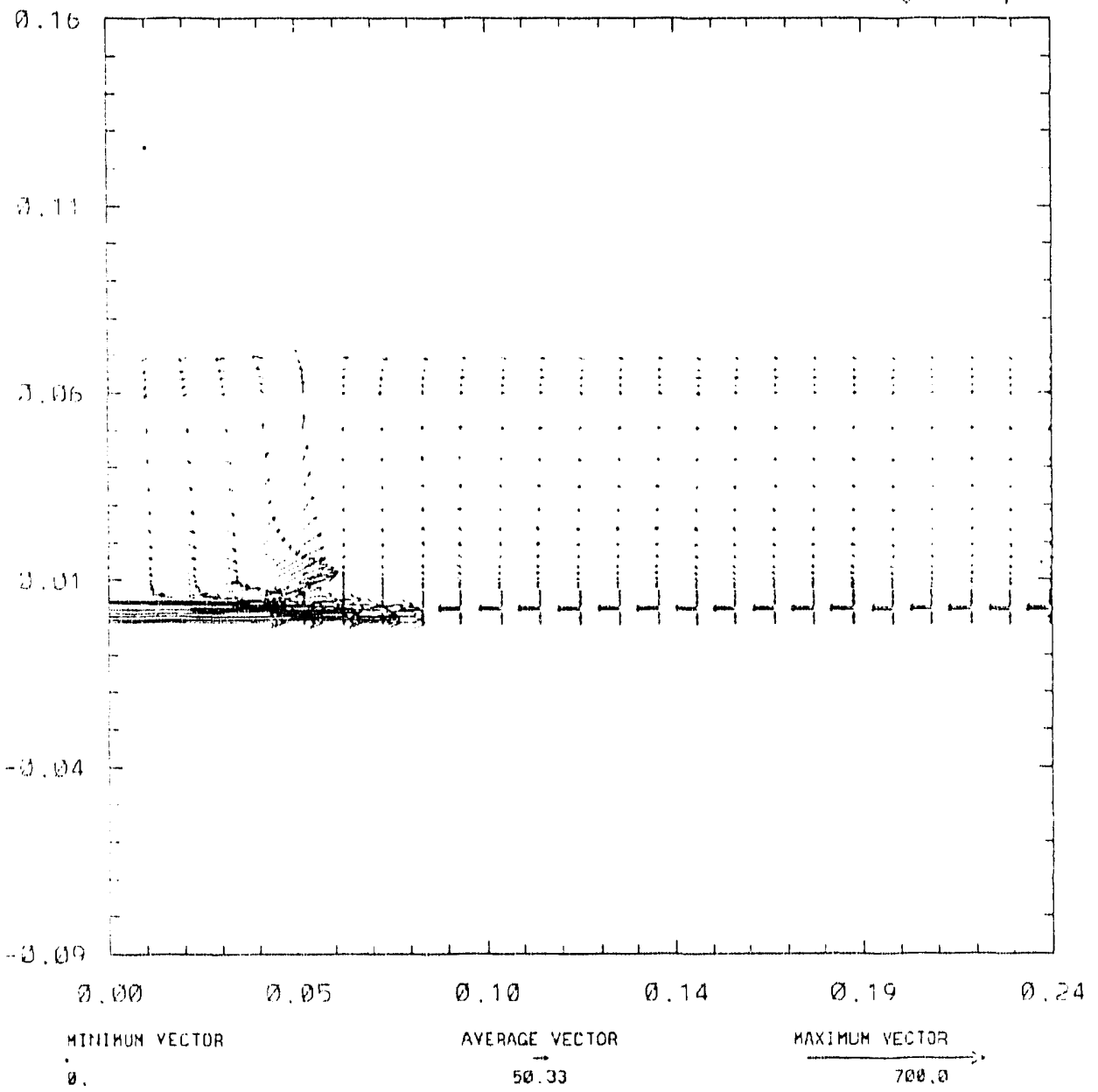


If the value of the loguni parameter is one (1), vectum will set the vector shaft scaling to logarithmic scaling (see Figure 6). Logarithmic scaled vectors have a logarithm $(\log (x)$ ) applied to each vector length.

FIGURE 6. Logarithmic vector shaft scaling

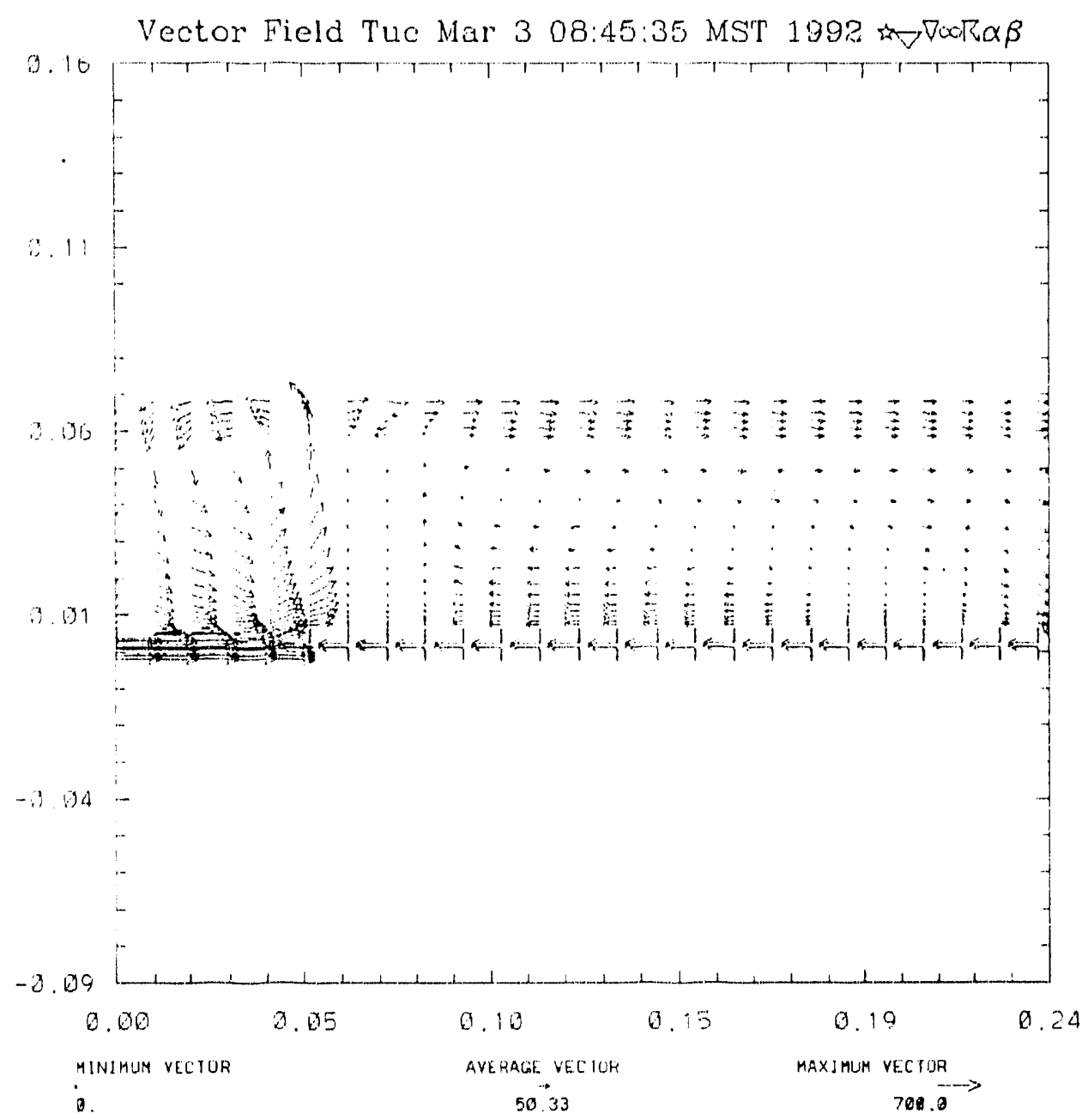

\subsubsection{Frac parameter}

The frac parameter is the float/integer toggle that determines the type of rounding that is used when the window viewport is automatically maximized $/ \mathrm{minimized}$. The frac parameter is of type integer. Note the following example:
1 it Erac
(o) max/min win int
(1) $\max / \mathrm{min}$ win Eloat: 
If the value of the frac parameter is zero $(0)$ and the $x$ min/.umax or ymin/ymax parameter is set to automatically maximize/minimize the window, the viewport will be zoomed to the next largest integer that is outside of the vector field.

If the value of the frac parameter is one (1) and the $x \mathrm{~min} / \mathrm{xmax}$ or $y$ min/ymax parameter is set to automatically maximize/minimize the window, the viewport will be zoomed to the nearest floating point value that is outside of the vector field.

\subsubsection{Flex parameter}

The flex parameter is the toggle that controls whether the $\mathrm{x}$ and $\mathrm{y}$ axis is scaled with respect to each other or independently of one another. The flex parameter is of type integer. Note the following example:

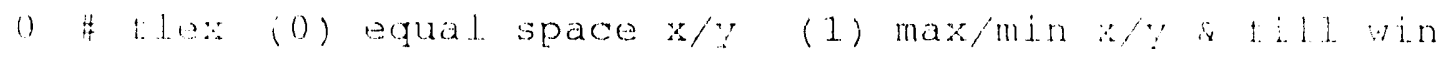

If the value of the flex parameter is zero (0), the viewport will be scaled so that the units in the $x$ direction will be equal in length $t(0)$ the units in the $y$ direction. Sec Figure 7.

FIC U/RE 7 . ligual unit scaling in the $x$ and $y$ directions

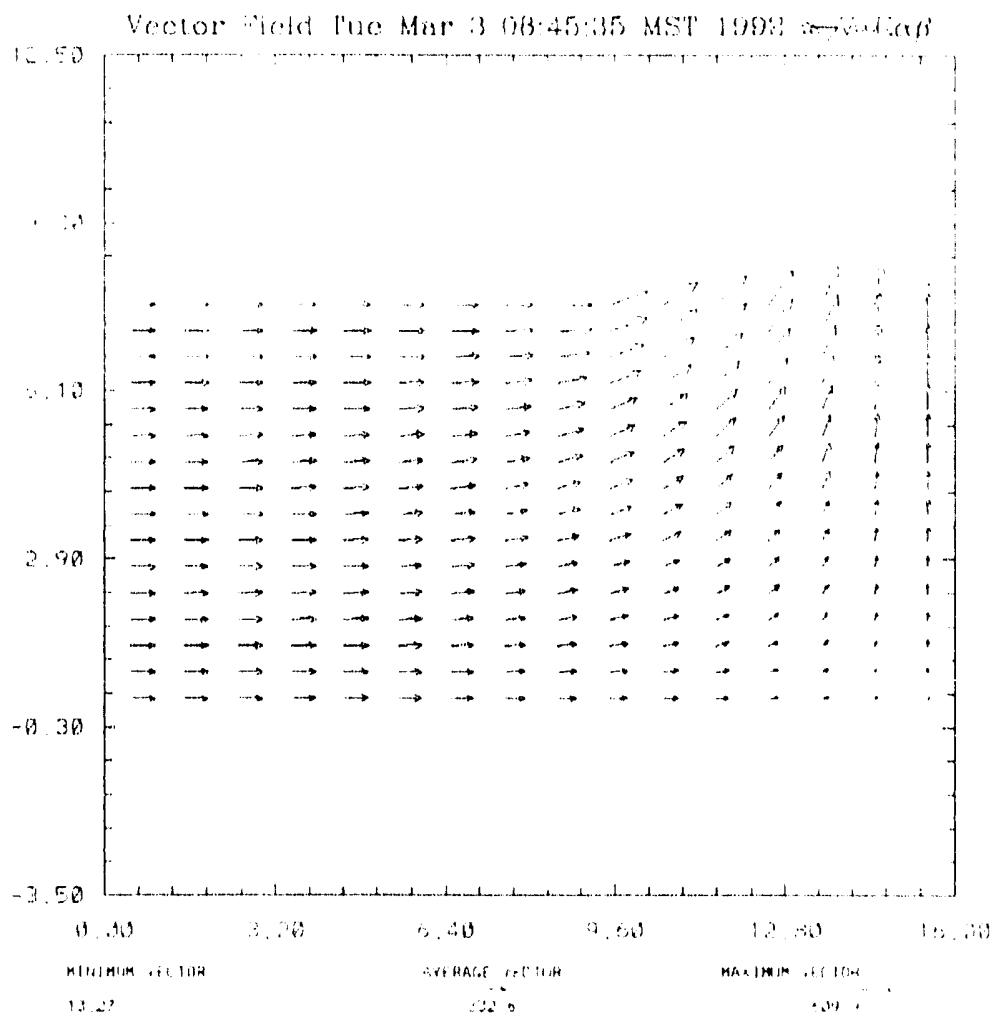


If the value of the flex parameter is one (1), the viewport will be scaled so that the units in the $\mathrm{x}$ and $\mathrm{y}$ direction will depend upon the outer extent of the vector field. If the vector field extends farther in the $x$ direction than the $y$ direction, for example, the units in the $x$ direction will be closer together than the y direction. The axis scale "flexes" to accommodate the maximum unit value in $x$ and $y$ direction. Changing the ax is independently forces vector dattit to fill the entire viewport window. See Figure 8.

FICiURE 8 . Independent $x$ and $y$ scaling

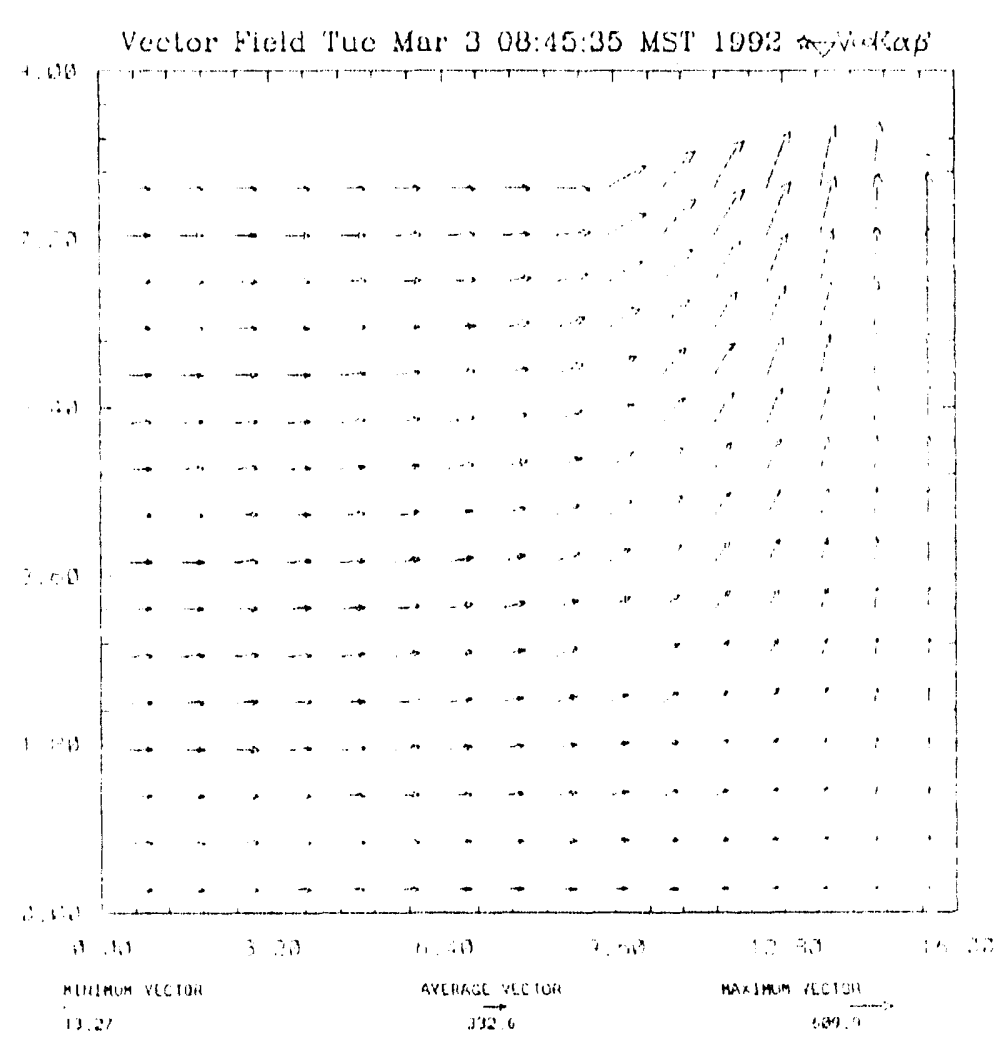

Scaling the $\mathrm{x}$ and $\mathrm{y}$ axis with respect to the data field and independently of each other maly not be appropriate for unit scaled vectors.

\subsubsection{Useaxs parameter}

The useavs parameter is the toggle that turns the display of the axis off and on. The axis includes the tickmarks, and the axis values. The useaxs parameter is of type integer. See Figure 1 , on page 8 for an illustration of the axis on a viewport window. Note the following 
example:
1 \# useaxs
(0) axis not displayed
(1) display axis

If the value of the usears parameter is zero (0). the axis will not be displayed. If the value of the useaxs parameter is one (1), the axis will be displayed.

\subsubsection{Useleg parameter}

The useleg parameter is the toggle that tums the display of the legend ofi and on. See Figure 1, on page 8 for an illustration of the legend on a viewport window. The value of the useleg parameter is of type integer. Note the following example:
1 \# useleg
(0) Legend not display
(1) Cisplay legend

If the value of the useleg parameter is zero (0), the legend will not be displayed. If the value of the useleg farameter is one (1), the legend will be displayed.

\subsubsection{Theta parameter}

The theta parameter is the angle (in degrees) between the vector shaft and the hypotenuse of arrow leg on line arrowheads. See Figure 9 for an illustration of the line arrowhead parameters. The theta parameter is of type lloat. Note the following example:

$$
30.0 \text { \# theta deg angle between rec shft \& arrow hypot lea }
$$

This parameter is ignored when character arrowheads have been specitied.

\section{FI(IURE 9. Line arrowhead parameters}

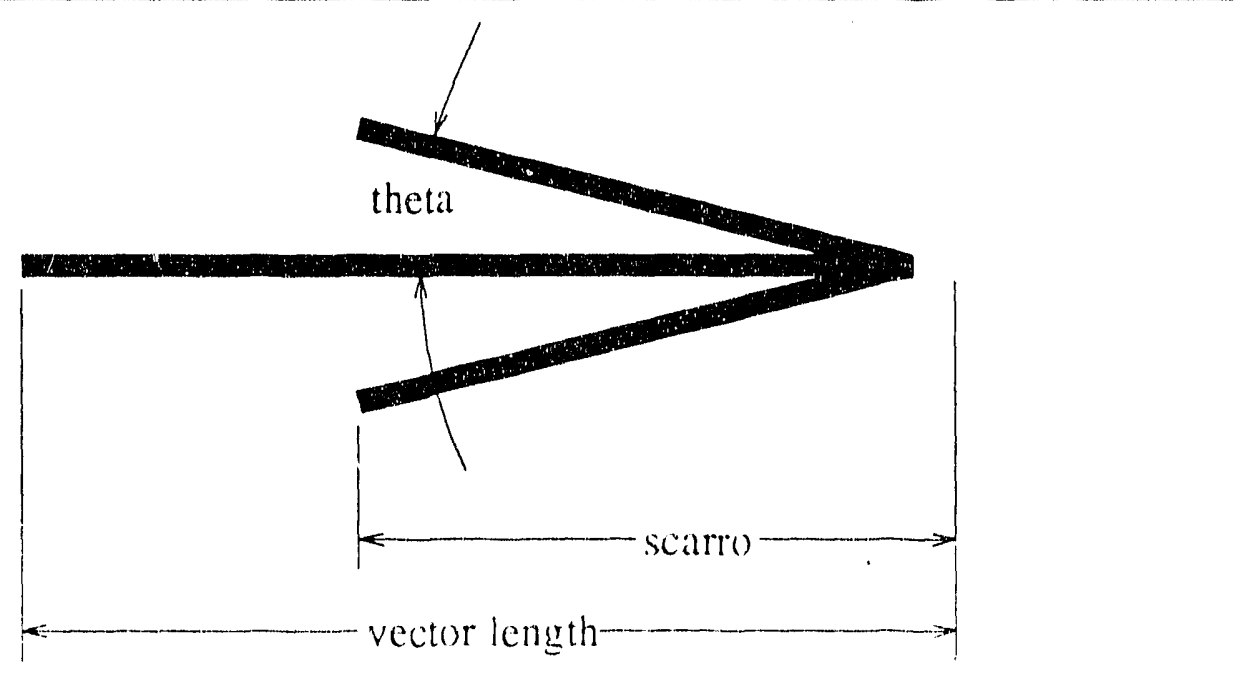




\subsubsection{Scarro parameter}

The scarro parameter is the height of the line arrowhead. The value of this parameter is defined as a percentage derived by the ratio between the triangle height of the arrowhead and the vector length. To specify an arrowhead half as big as the vector length, for example, the scarro parameter would be fifty percent (50). The scarro parameter is of type integer. See Figure 9, on page 17, for an illustration of the line arrowhead parameters. Note the following example:

15 \# scarro arrow len (triangle height) is of vec shet

This parameter is ignored when chaluater anowheads have been specified.

\subsubsection{Shfmin parameter}

The shfimin parameter is the minimum vector length to draw line arrowheads. Line arrowheads will ot be drawn on vectors that have a shorter length than the shfinin parameter. See Figure 9, ar page 17 for an illustration of the line arrowhead parameters. The shfimin parameter is of type lloat. Note the following example:
0.2
\# shtin
minimum shat size for arrowheads

This parameter is ignored when character arrowheads have been specified.

\subsubsection{Altchr parametter}

The altchr parameter is a three way toggle that specifies the arrow head type. The altohr parameter is of type integer. Note the following example:

$$
0 \text { \# altchr (-1) line arrow (0) norm char (1) extend char }
$$

If the value of the altchr parameter is negative one $(-1)$, line arrowheads will be drawn. See Figure 18 , on page 27 for an example vector field with line arrowheads.

If the value of the altchr parameter is zero (0), normal character (single stroke) arro wheads will be drawn and vectum will use the character specified by the arwchr parameter (see Section 2.1.25, on page 22).

If the value of the altchr parameter is one (1), high quality character (i.e. double stroke) arrowheads will be drawn and vectum will use the character specified by the aruchr parameter (see Sertion 2.1.25, on page 22). This option also allows an extended character set to be used for arowheads. The extended character set lets the user take advantage of 
numerous symbols. Sce Figures 1()-13, on pages 19 and 22 for a displaty of the symbols available.

\section{FI(iURE 10. Alternate character set}

PLCHHQ - COMPLEX CHARAC'TER SEI'

FUNCTION CODES SPECIFYING SIZE, FONT, MVD (

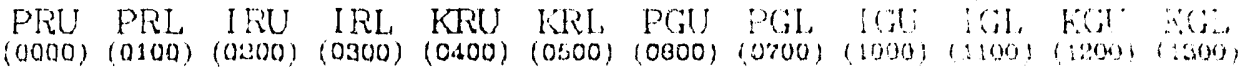

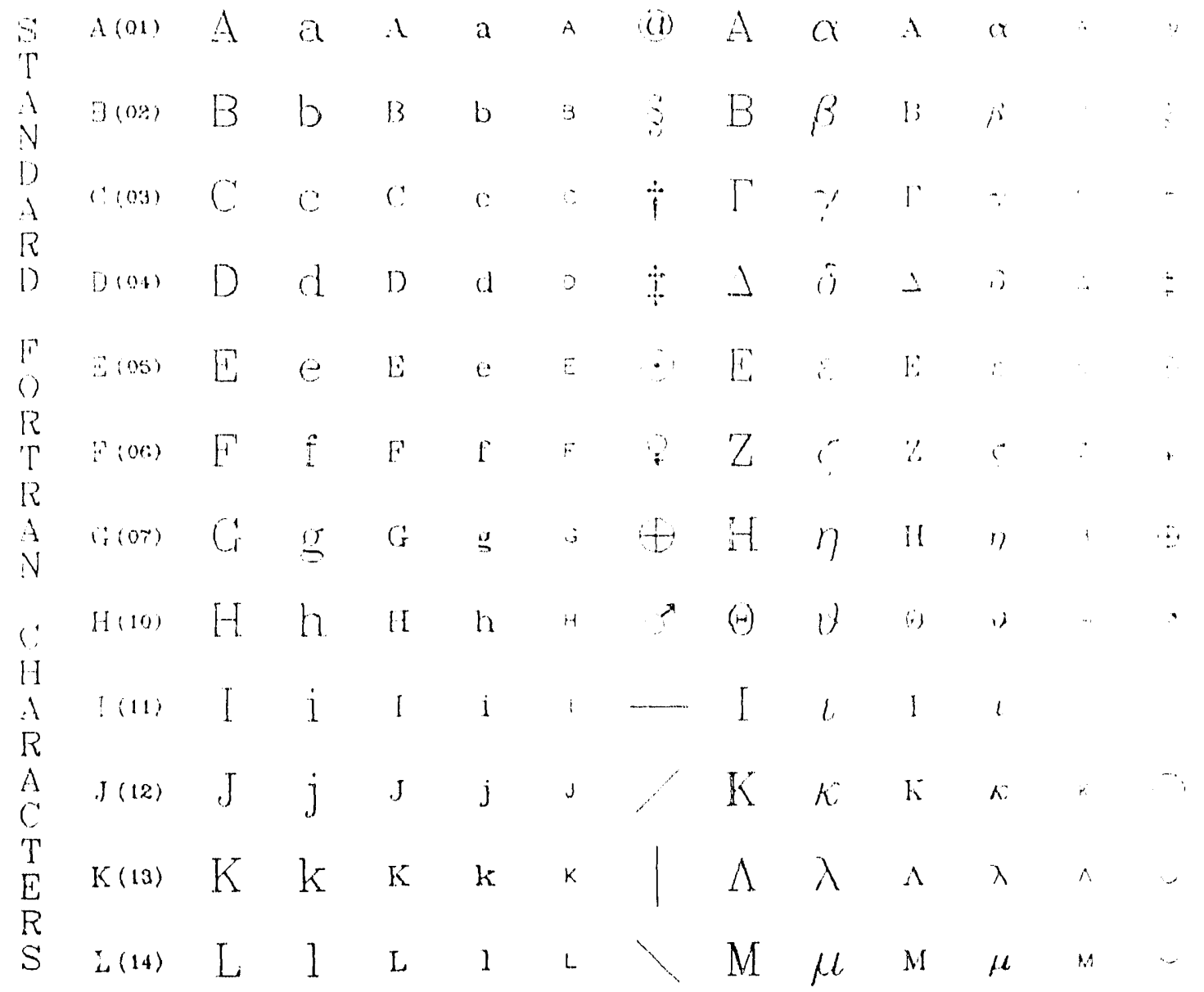


Voum Users cinide

HIGUKl: 11. Altermate character set (cont.)

\section{PLCHHQ - COMPLEX CHARACTER SET}

FUNCTION CODES SPECIFYING SIZE, FONT, AND CASE

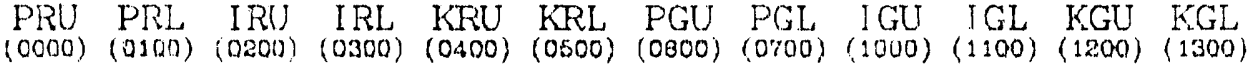

$\mathrm{S} M(15) \mathrm{M} m \mathrm{M} \mathrm{m} \quad \mathrm{M}-\mathrm{N} v \mathrm{~N} v \mathrm{~N}$,

$\begin{array}{llllllllllll}A & N\{B\} & N & N & N & n & N & - & \Xi & \xi & \xi\end{array}$

A $0: 1 \%: 0$ O 0 O

R

D $3:(m)$

F Q in

R

$\mathrm{T}$

R(s) R

R

i $3(23) \& 8$

$\therefore \mathrm{T}, \mathrm{T}, \mathrm{T}$ t

$\mathrm{H}$

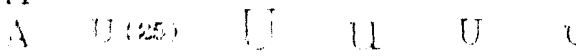

R

$\therefore \because \% V \quad V \quad V$

T $W(27) \quad W \quad W \quad W \quad W$

$\mathrm{R}$

$\mathrm{S} X(3), \mathrm{X} \quad \mathrm{X} \quad \mathrm{X} \quad \mathrm{x}$




\section{PLCHHQ - COMPLEX CHARACTER SET}

FUNCTION CODES SPECIFYING SIZE, FONT, AND CASE

PRU PRL IRU IRL KRU KRL PGU PGL IGU IGL KGU KGL

$\begin{array}{lllllll}S & Y(31) & Y & Y & Y & y\end{array}$

$\begin{array}{llllllll}\mathrm{A} & \mathrm{Z}(32) & \mathrm{Z} & \mathrm{Z} & \mathrm{Z} & \mathrm{z} & \mathrm{z} & \mathrm{V}\end{array}$

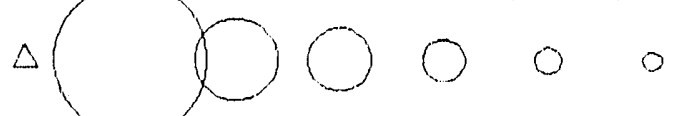

$\mathrm{N}$

D

A

$R$

$0(33) \quad 0$

L) $\quad L(34)$

1

F

()

$\underset{\mathrm{T}}{\mathrm{T}}$

$\mathrm{T}$

A

C

$\mathrm{H}$

$A B(+1) 6 \div 6 \div 5 \Rightarrow 6 \times 6 ; \Rightarrow 6$

R

A $r(+2) \quad r \equiv 7 \equiv$

$\mathrm{C}$

$\mathrm{T} 8(45) 8<8<8 \perp 8,8,{ }_{8} 8$

$\mathrm{R}$

S

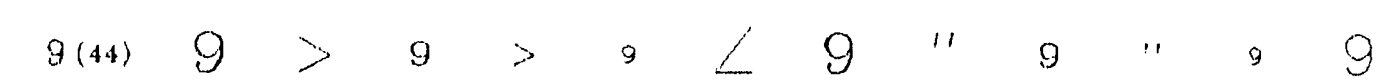




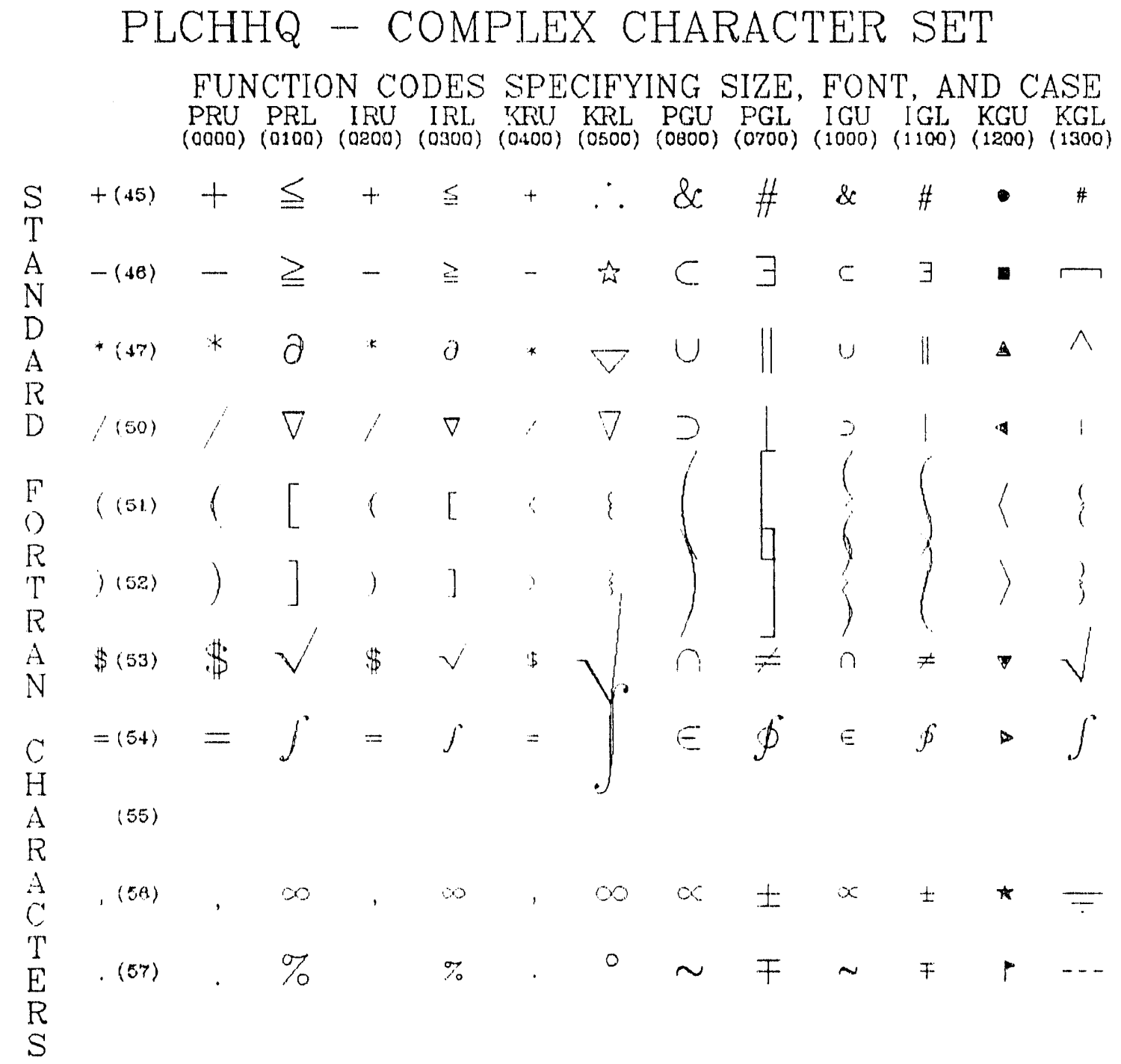

\subsubsection{Arwchr parameter}

The arwchr parameter specifies the arrowhead character. The arwchr parameter is of type character string. Note the following example:

$$
\text { 'v' \# arwchr arrowhead character }
$$


The type of arrowhead displayed will depend upon the value of the altchr parameter (see Section 2.1.24, on page 18). If normal character type is specified by altchr parameter, an arrowhead with a single stroke character will be drawn. If extended character type is specified by altchr parameter, double stroke characters with serifs will be used for the arrowheads.

The extended character set allows the user to take advantage of numerous symbols. See Figures 1()-13, on pages 19 and 22 for a display of the symbols available.

To use an altemate character symbol, observe the following procedure:

1. Lociate the desired symbol from Figures 10-13.

2. Determine the symbol's three character function code family as indicated at the top of the column that the symbol is contained in.

3. Prefix the function code with the grave symbol (").

4. Determine the symbol's one character standard fortran character family as indicated at the left side of the row that the symbol is contained in.

5. Prefix the standard fortran character with the grave symbol (').

6. Concatenate the function code family and the fortran character fiamily.

To specify a double dagger $(+)$ symbol for the arrowhead character, you would use 'KRL for the function code family and ' $\mathrm{D}$ for the standard fortran character family or ' $\mathrm{KRT}$ ' $\mathrm{D}$

If more than one symbol from the same function code farnily (or same column) is used, the function code should be specified only once. In addition, the prefixed grave symbol(') can apply to multiple entries from the fortran character family. The following string is an exam. ple of how multiple symbols from the same function code family would be specified:

' PGL ' ABCDEFGHI JKIMNOPQRSTUVWX

This string would generate the symbols as displayed in Figure 14.

FIGURE 14. Characters in the same function code family

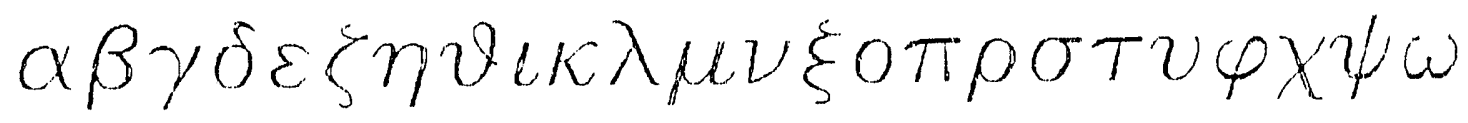

If symbols from different function code families are used, the function code should te specified each time. The following string is an example of how symbols from different function 
Vectum User's ciuide

code families would be specified:

'PRU' - 'PRL' - 'IRU' - 'IRL' - 'KRU' - 'KRL'- 'PGU'- 'PGL'-

This string would generate the symbols as displayed in Figure 15.

FIGURE 15. Characters in differing function code families

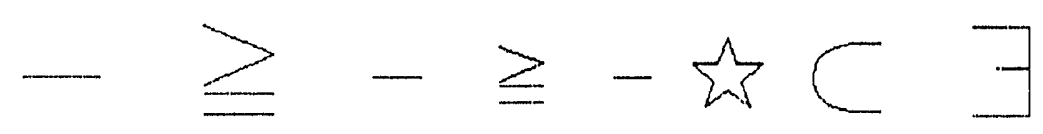

Alternate character symbols can also be used in the plot title (see Section 2.1.4, on page 5, regarding the "Title parameter") and are specified in the same manner.

\subsubsection{Arwsiz parameter}

The arwsiz, parameter controls the maximum size of the arrowhead character (see Section 2.1.25. on page 22, regarding the "Anvchr parameter"). The anvsiz, parameter is of type integer. Note the following example:

$$
20 \text { \# \#rwsiz maximum arrowhead size }
$$

Each arrowhead character is drawn at the size specified by this parameter when the useshf parameter (see Section 2.1.28, on page 25) is set to display a constant arrowhead size.

When the amvsiz, parameter is set to display variable arrowhead size (i.e. no vector shaft), the size of the each arrowhead character is dependant upon the magnitude of it's u/v value. The arrowhead characters are scaled from the smallest character size to the size specified by this parameter. The range of the anwsiz parameter is from 3 to 24 .

\subsubsection{Arwang parameter}

The arwang parameter is the angle of the arrowhead character (see Section 2.1.25, on page 22 , regarding the "Arwchr parameter"). The arwang parameter is of type integer. Note the following example:

0 \# arwang arrowhead angle

The range of the arwang parameter is from 0 to 360 . If the angle is set to zero, the arrowhead character is usually oriented to the vector shaft appropriately. 


\subsubsection{Useshf parameter}

The useshf parameter is a three way toggle for controlling the arrowhead scaling and turning .le vector shaft on and off. The useshf parameter is of type integer. Note the following example:
1 \# useshf
(-1.) scatter
(0) no shaft
(1) use shaft

If the value of the useshf parameter is negative one $(-1)$, the vector shaft will not be displayed and the arrowhead (or marker) size will not be scaled in proportion to the $u / v$ values. Instead, each arrowheald will be a constant value as specified by the arwsiz parameter (see Section 2.1.26, on page 24). See Figure 16 for a scatter plot with a constant marker size.

FIGURE 16. Scatter plot with a constant marker size

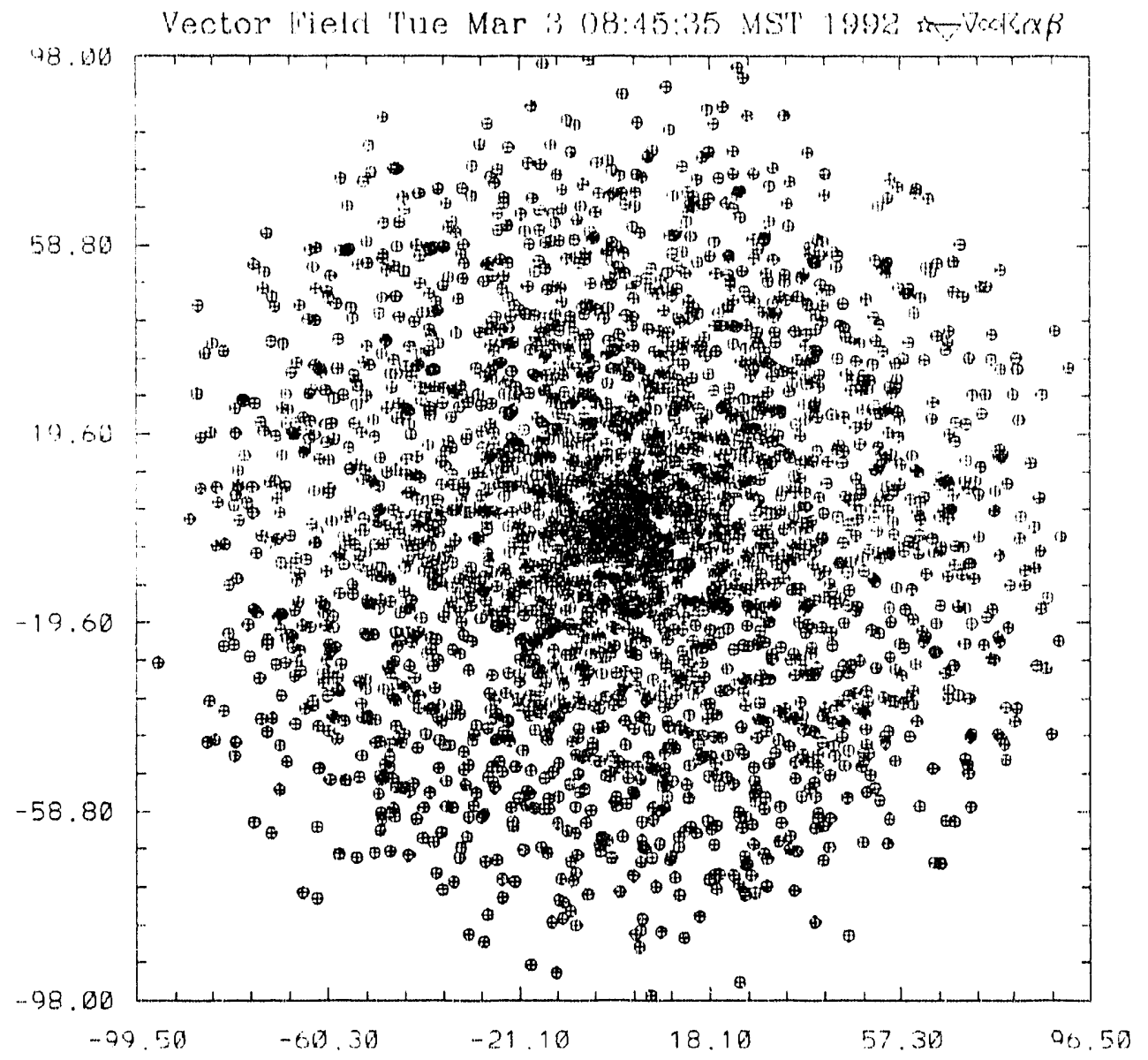


If the value of the useshf parameter is zero (0), the vector shaft will not be displayed and the arrowhead (or marker) size will be scaled in proportion to the u/v values. See Figure 17 for a scatter plot with a variable marker size.

FIGURE 17. Scatter plot with a variable marker size

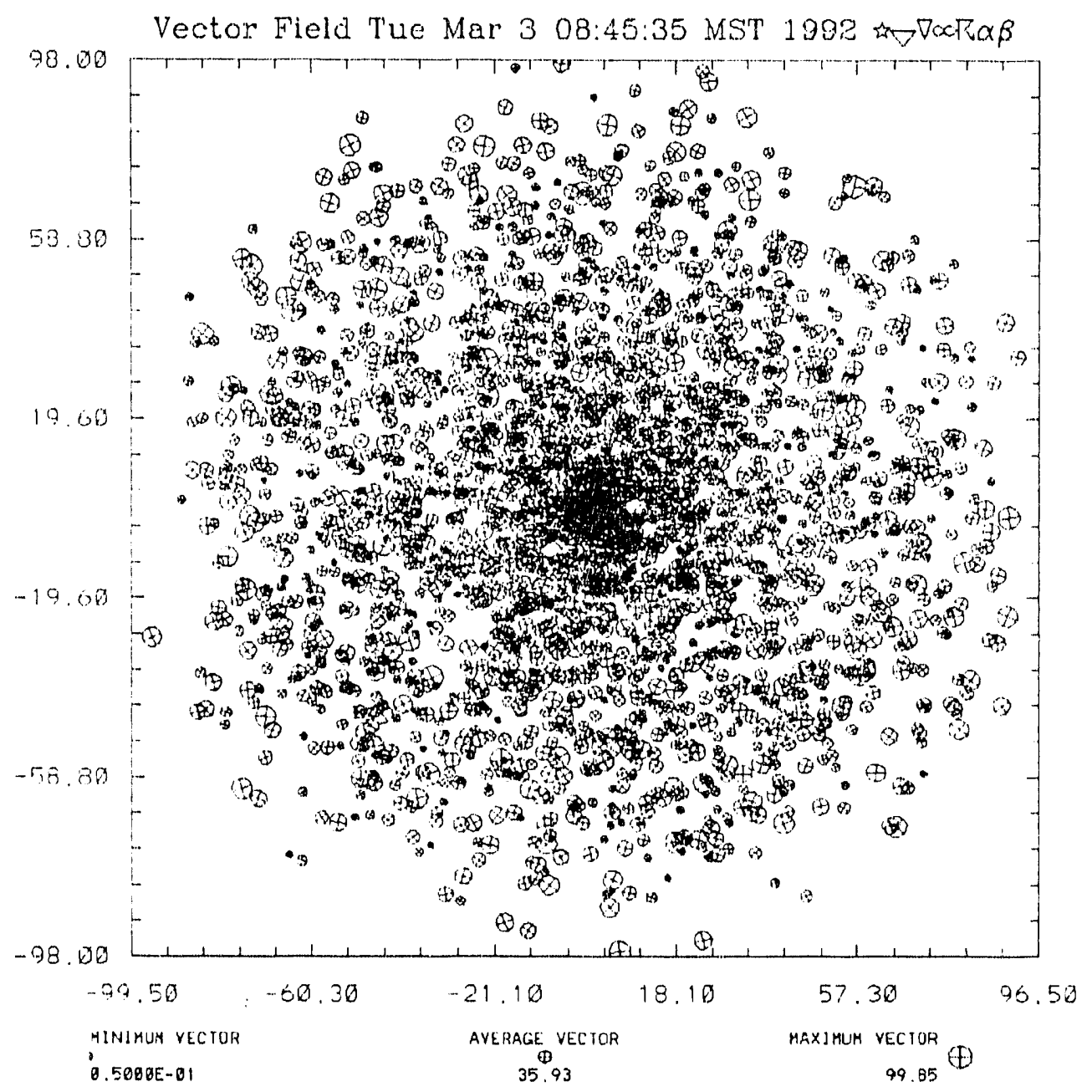

If the value of the useshfparameter is one (1), the vector shaft will displayed and the arrowhead size will be scaled in proportion to the $u / v$ values.

\subsubsection{Decinc parameter}

The decinc parameter is the decimation increment value. This parameter can be used to 
reduce the number of dataset values that are displayed. The decinc parameter is of type integer. Note the following example:

1 \# decinc decimation. if (decinc = 1) no values skipped

The decinc parameter decimates the data values while they are being read from the data file into vectum.

If the value of the decinc parameter is one (1), vectum will read every data value (see Figure 18).

\section{FIGURE 18. Line arrowheads with decimation factor of 1}

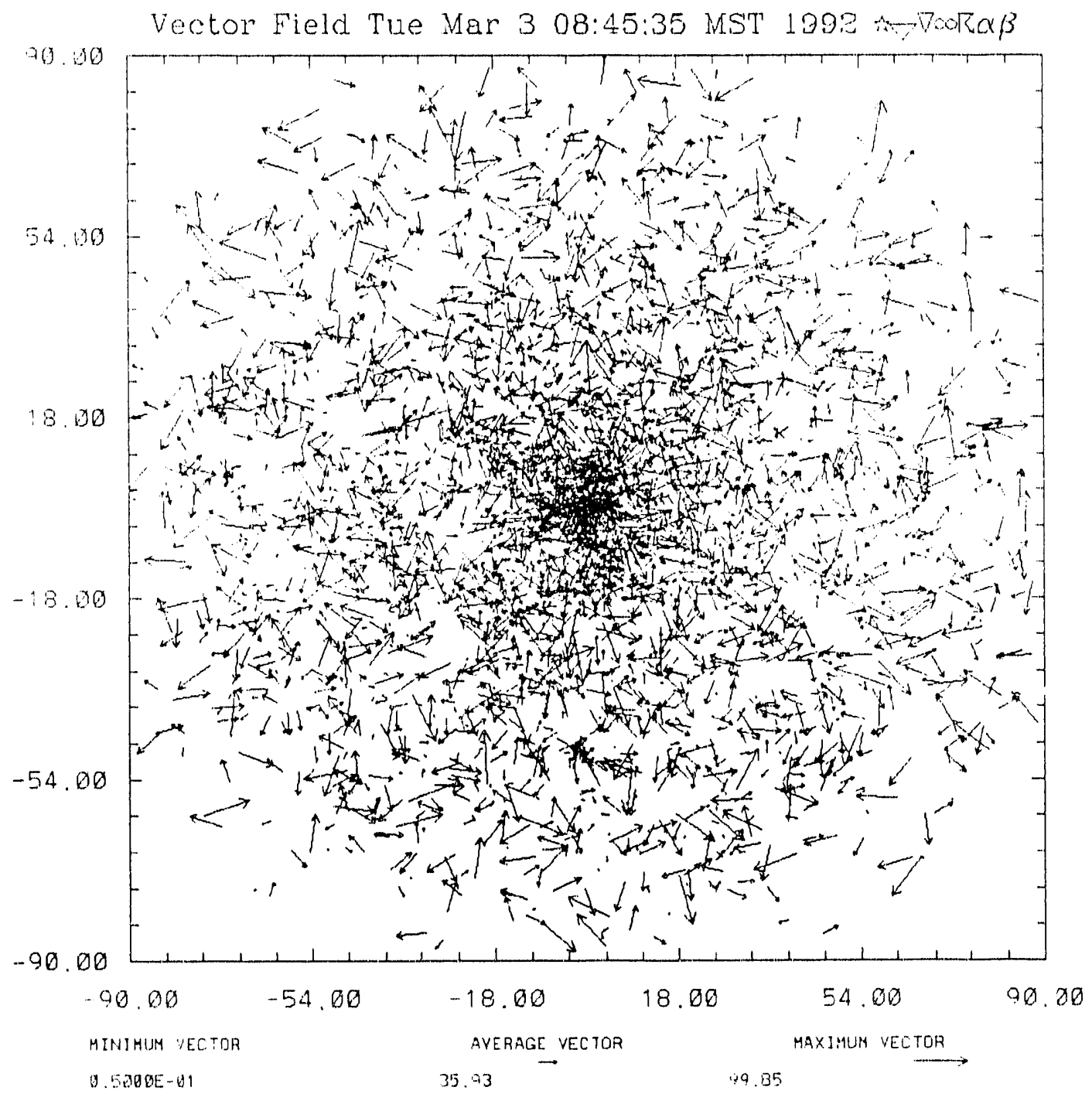


If the parameter is five (5), for example, every fifth value will be read (see Figure 19). The decinc parameter is used internally in modulus division to determine even multiples of the dataset values to be displayed.

FIGURE 19. Line arrowheads with decimation factor of 5

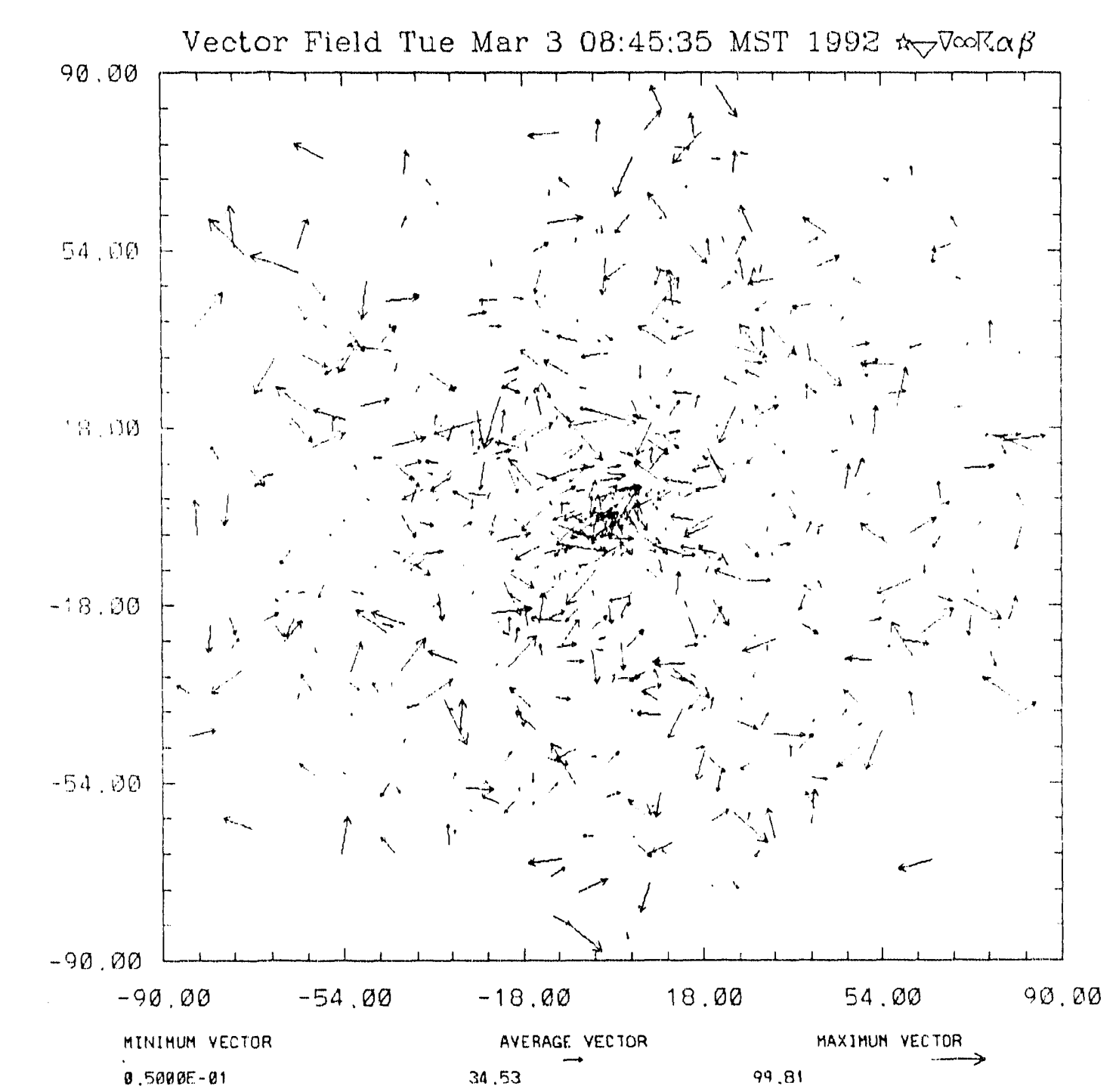




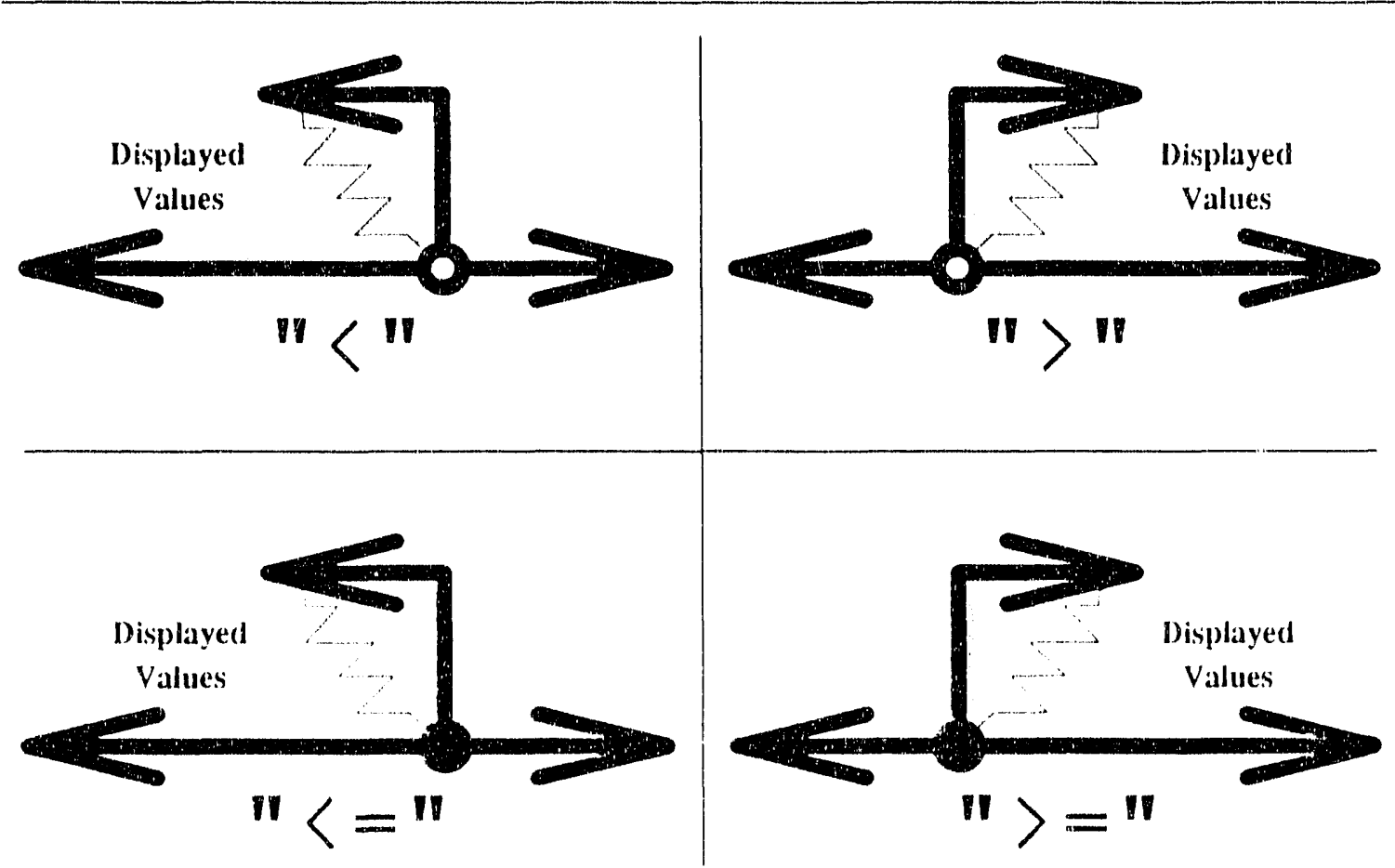

\subsubsection{Vecmin parameter}

The vecmin parameter is the value of the minimum vector length displayed. The vecmin parameter is of type float. Note the foilowing example:

$$
0.0 \text { \# recmin minimum rector length aisplayed }
$$

This parameter works in conjunction with the optmin parameter (see Section 2.1.31). If the user wanted to display only those vectors with a length greater thar zero, for example, the vecmin parameter would be zero $(0.0)$ and the optmin parameter would be the greater than (' > ') logical operator. See Figure 23, on page 32 for an illustration specifying a maximum range with the vecmin and optmin parameter.

\subsubsection{Optmin parameter}

The optmin parameter uses a logical operator to specify the minimum range of vectors displayed. The optmin parameter is of type character string. Note the following example:

$$
\text { '?' \# optmin display vec }(s,>, \iota=>===\text {, ?) min len }
$$


If the optmin parameter is the less than $\left({ }^{\prime}<\right.$ ') logical operator, only vector lengths less than (but not including) the value specified by the vecmin parameter will be displayed.

If the optmin parameter is the greater than ('>') logical operator, only vector lengths greater than (but not including) the vallue specified by the vecmin parameter will be displayed.

If the optmin parameter is tho less than or equal to $(1<=1)$ logical operator, only vector lengths less than the value sfucified by the vecmin parameter will be displayed. This logical operator also includes the vecmin value itself in the range.

If the optmin pitrameter is the greater than or equal to (' $\left.>={ }^{\prime}\right)$ logical operator, only vector lengths greater than the value specified by the vecmin parameter will be displayed. This logicall operator also includes the recmin value itself in the range.

If the optmin parameter is the equal to $(1==1)$ logical operator, only vector lengths eyual to the value specified by the vermin parameter will be displayed.

If the optmin parameter is a question mark (' ?') symbol, the vecmin parameter is ignored.

See Figure 20, on page 29 for an illustration that shows using the logical operators to specify a range.

\subsubsection{Vecmax parameter}

The vecmax parameter is the value of the maximum vector length displayed. The vermax parameter is of type float. Note the following example:

$$
0.0 \text { \# vecmax maximum rector length displayed }
$$

This parameter works in conjunction with the optmax parameter (see Section 2.1.33). If the user wanted to displaty only those vectors with a length less han twenty-one hundred, for example, the vecmax parameter would be twenty-one hundred $(2100.0)$ and the optmax parameter would be the less than ('<') logical operator. See Figure 22, on page 32 for an illustration specifying a minimum range with the vecmax and optmax parameter.

\subsubsection{Optmax parameter}

The optmax parameter uses a logical operator to specify the maximum range of vectors displayed. The optmax parameter is of type character string. Note the following example:

$$
\text { '?' \# optmax display rec }(\because, \therefore,<=, \therefore=,==\text {, ?) max len }
$$


If the optmax parameter is the less than $('<1)$ logical operator, only vector lengths less than (but not including) the value specified by the vecmax parameter will be displayed.

If the optmax parameter is the greater than ('>') logical operator, only vector lengths greater than (but not including) the value specified by the vecmax parameter will be displayed.

If the optmax parameter is the less than or equal to $(1<=1)$ logical operator, only vector: lengths less than the value specified by the vecmax parameter will be displayed. This logical operator also includes the vecmax value itself in the range.

If the optmax parameter is the greater than or equal to $(1:=1)$ logical operator, only vector lengths greatter than the value specified by the vecmax parameter will be displayed. This loggical operator also inc/udes the vecmax value itself in the range.

If the optmax parameter is the equal to $\left({ }^{\prime}==1\right)$ logical operator, only vector lengths equal 10) the value specified by the vecmax parameter will be displayed.

If the optmal parameter is a question mark('?') symbol, the vecmax pallameter is ignored.

See Figure 20, on page 29 for an illustration that shows using the logical operators 10 specify a range.

The optmin and optmax parameters can be used together to specify a linite range of displayed vectors. See Figure 21.

FIC:URE 21. Finite range specified

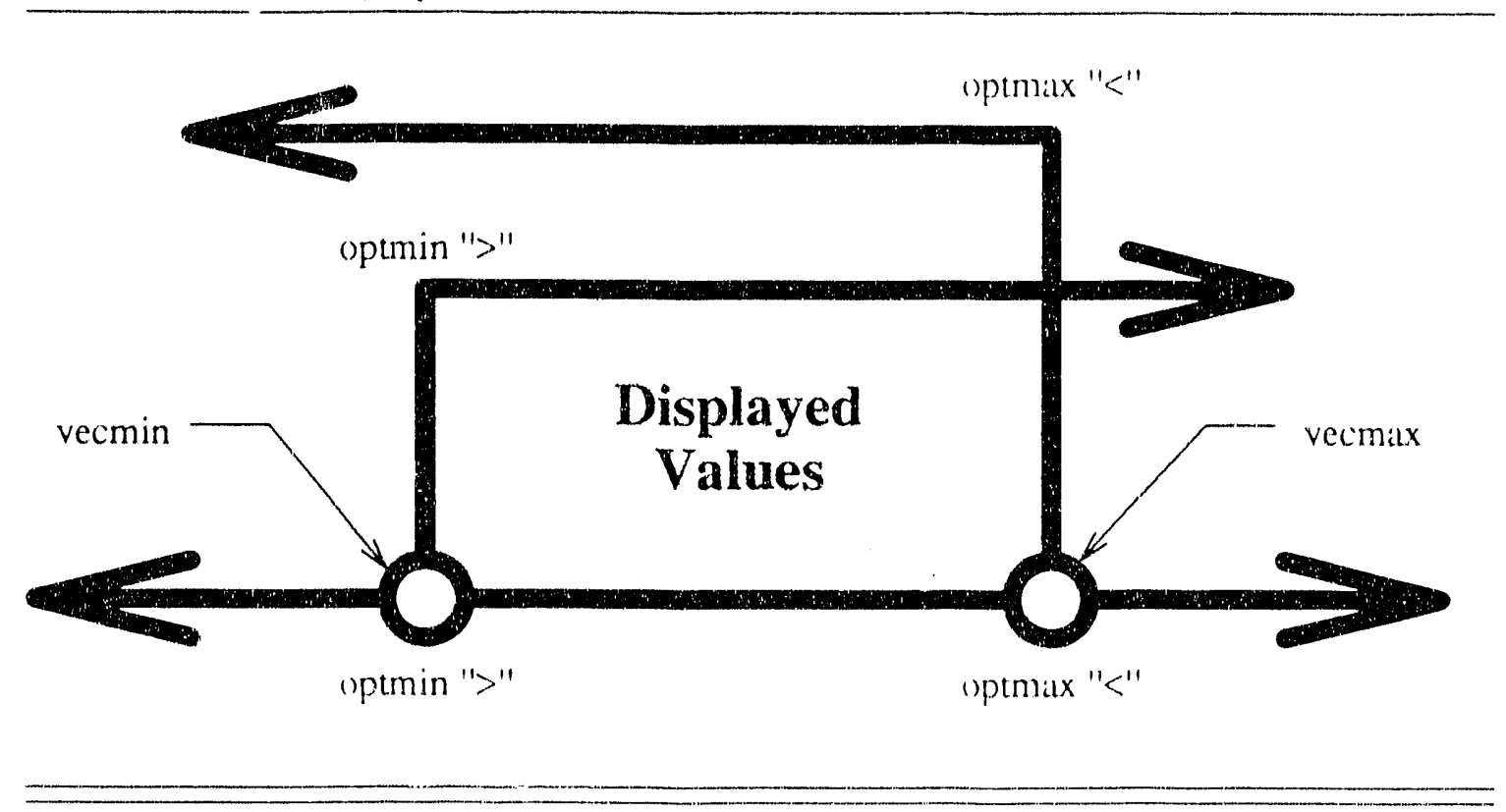



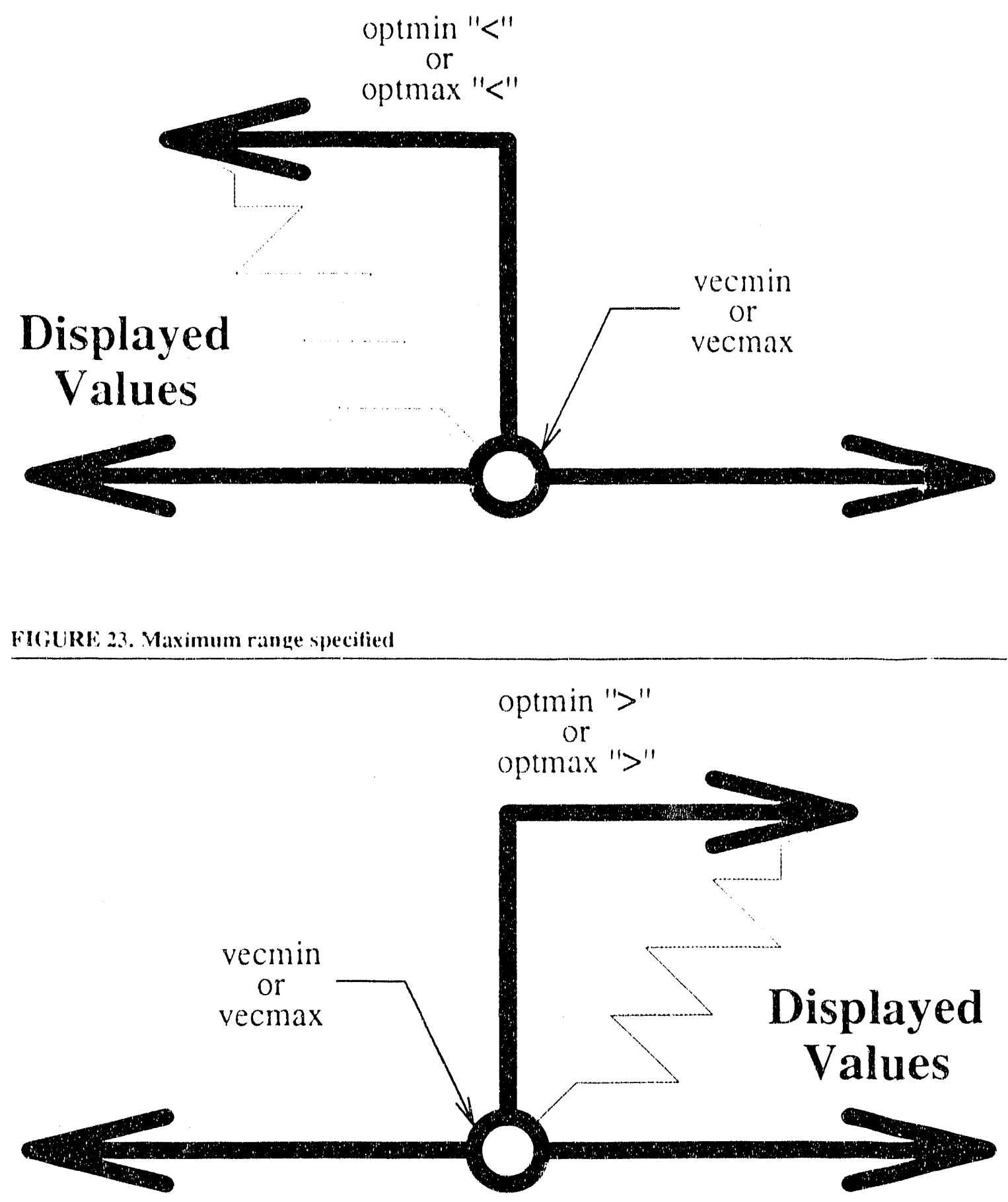
The optmin and optmax parameters can also be used together to specify a infinite range of displayed vectors. See Figure 24.

FIGURE 24. Infinite range specified

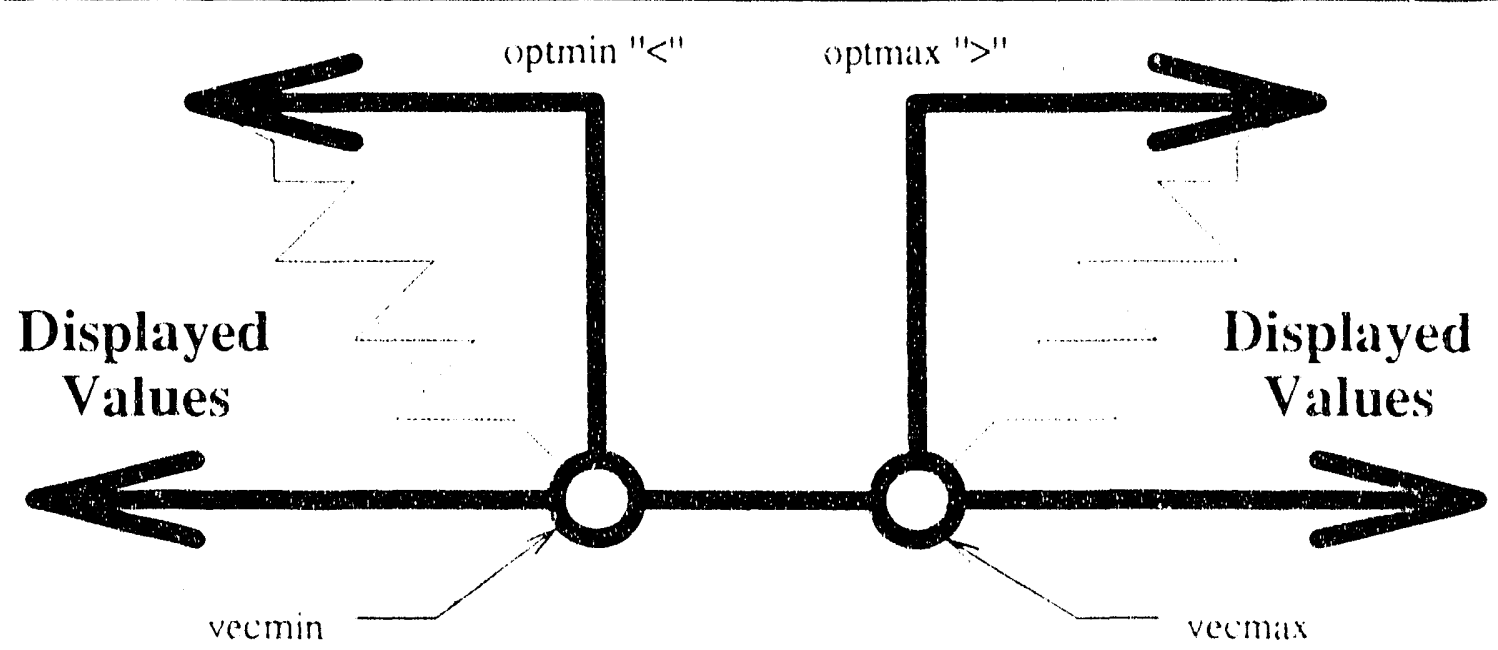

\subsubsection{Movie parameter}

The movie parameter is a toggie that allows the user 10 specify multiple titenames on the command line and use multiple datasets to build a movie or merge several frames toggether. The movie parameter is of type integer. Vote the collowing example:
1. it movie
(i) merge muiti trume
(1) xppond :mat: : : am

This parameter is not currently implemented.

\subsection{Geometry database}

Vectum also gives the user the capability to define simple, 2D geometry that is displayed with the vector field. The filename is specified by the filge'o parrameter (see Section 2.1.8. on page 6) in the parameter file.

The format of the ASCII database and the geometry descriptors are described in Sections 2.2.1-2.2.6 on pages 36-41. The following is an example listing of a geometry database:

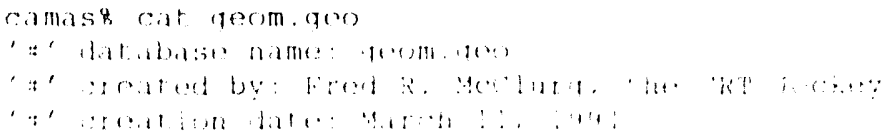


Yectum User's Guide

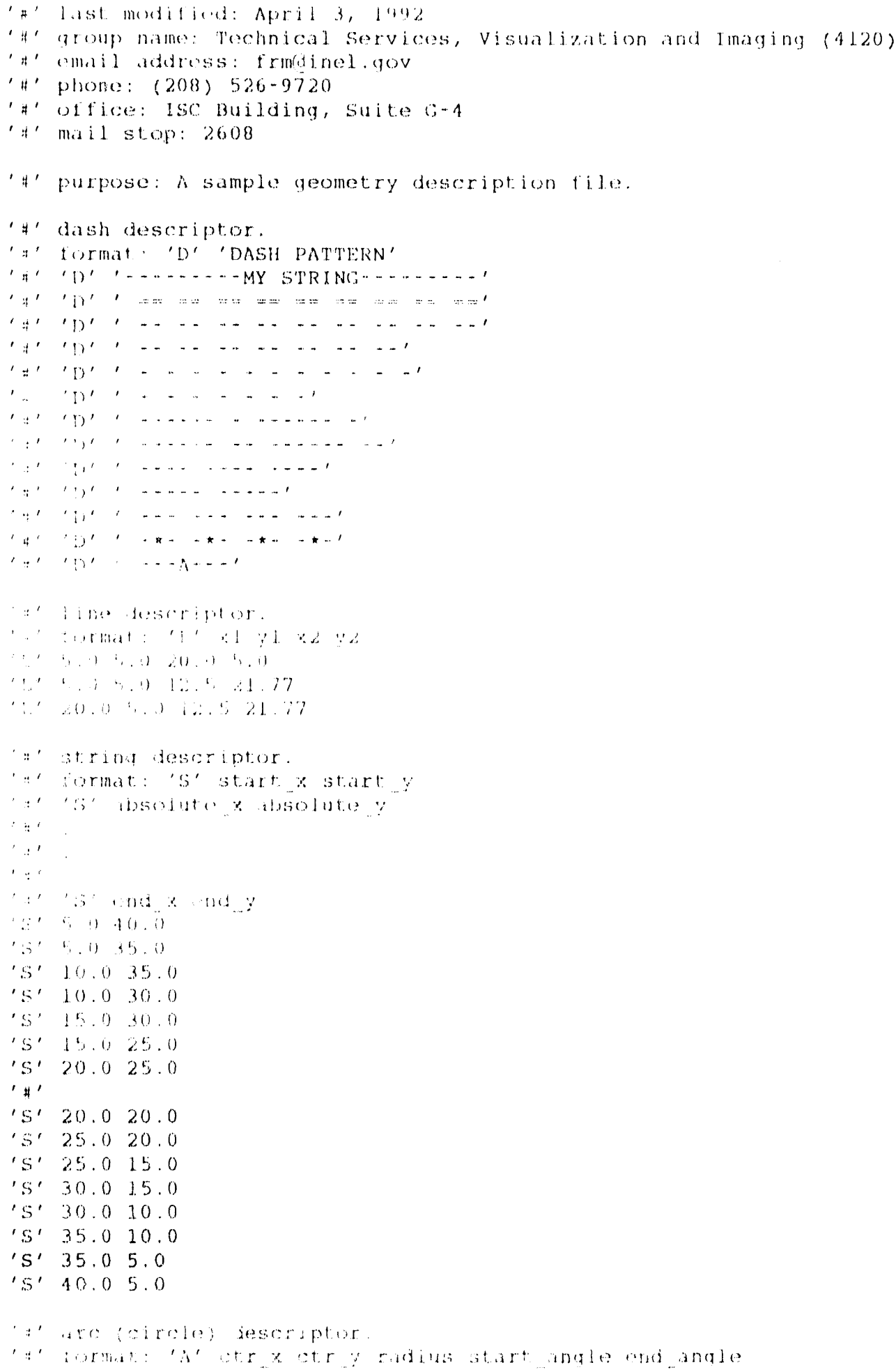




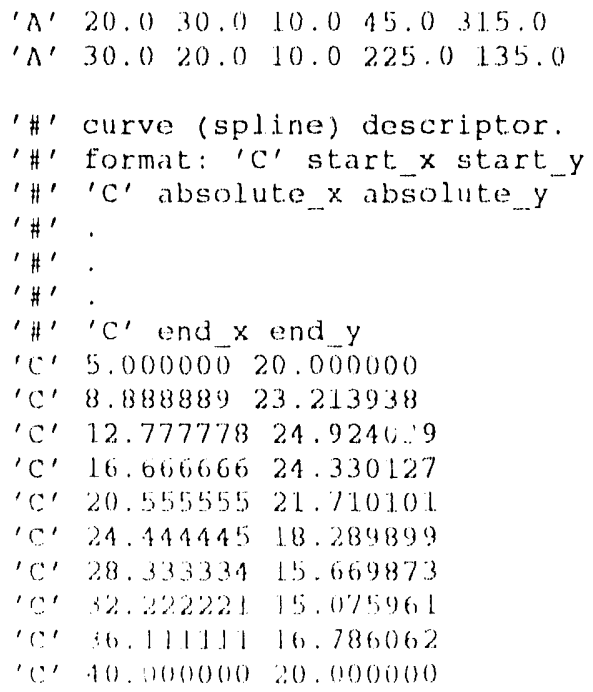

Figure 25 is was generated using the geometry database listed above.

FICURE 25. Miscellaneous geometry defined by the geometry database

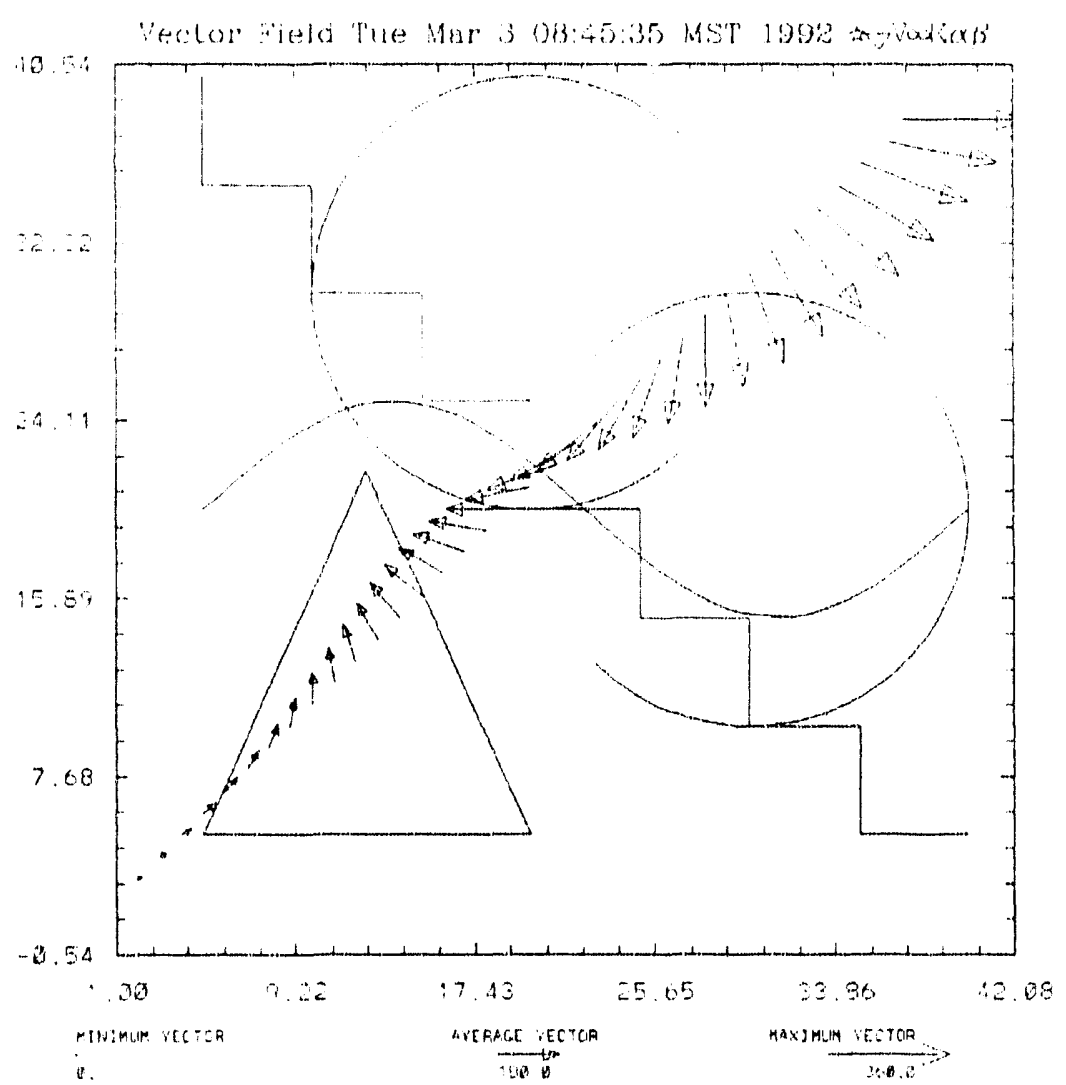




\subsubsection{Comment descriptor}

Comment lines can be used in the geometry database to assist in documentation. Each record that begins with '\#' denotes a comment. Any thing following the '\#' is ignored. Note the following example:

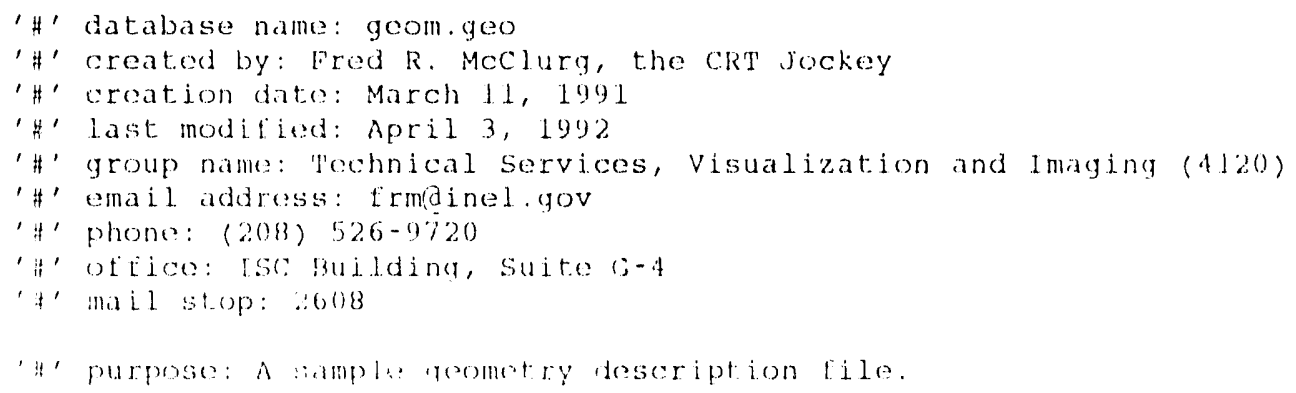

\subsubsection{Dash descriptor}

The dash descriptor is used to change the line (or carve) style from a solid to at user specified pattern. A dash descriptor begins with ' $\mathrm{D}$ ' and is followed by at chartacter string enclosed in single yuotes $\left({ }^{\prime}\right)$. The character string specifies the dash paltern of the line style on all geometry defined atter the dash descriptor is specified. Note the following example:

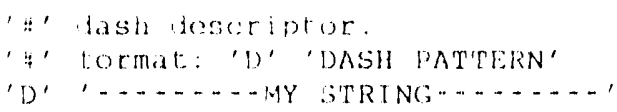

Figure 26, on page 37 wals generated by using several different dash descriptors in a geometry database and a vector field. 
FICURE 26. Geometry drawn using the dash descriptor

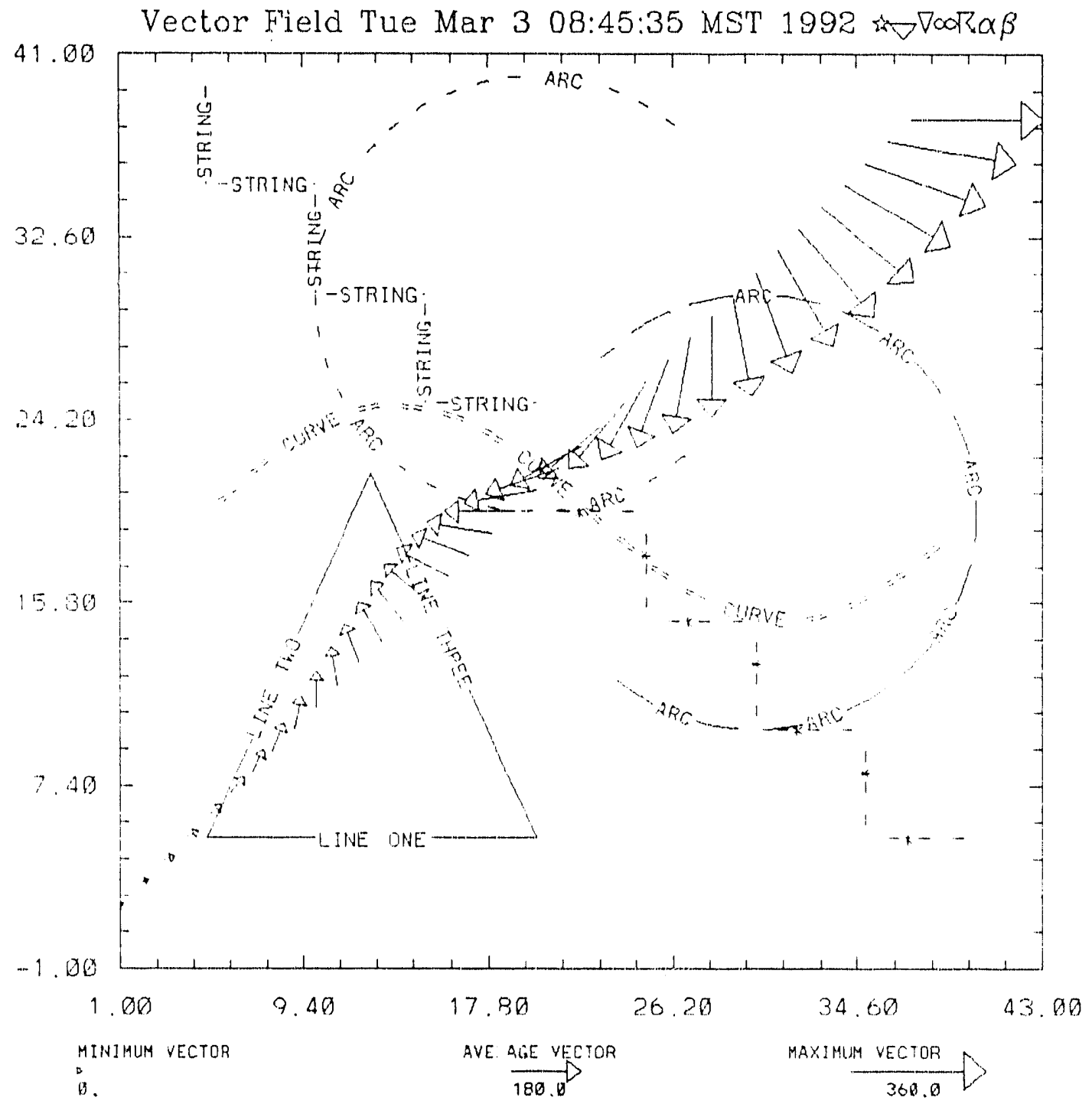

\subsubsection{Line descriptor}

The line descriptor defines a line by specifying end point coordinate values. A line descriptor begins with ' $\mathrm{L}$ ' and is followed by four floating point values. Note the following example:

\# line descriptor.

'\#' tormat: 'L' $x !$ y 1 x $y^{2}$

'L' 5.05 .020 .150 
The mesh displayed in Figure 27 wats generated by using the line descriptor.

FIC:URE 27. Triangular grid mesh drawn using the line descriptor ${ }^{1}$

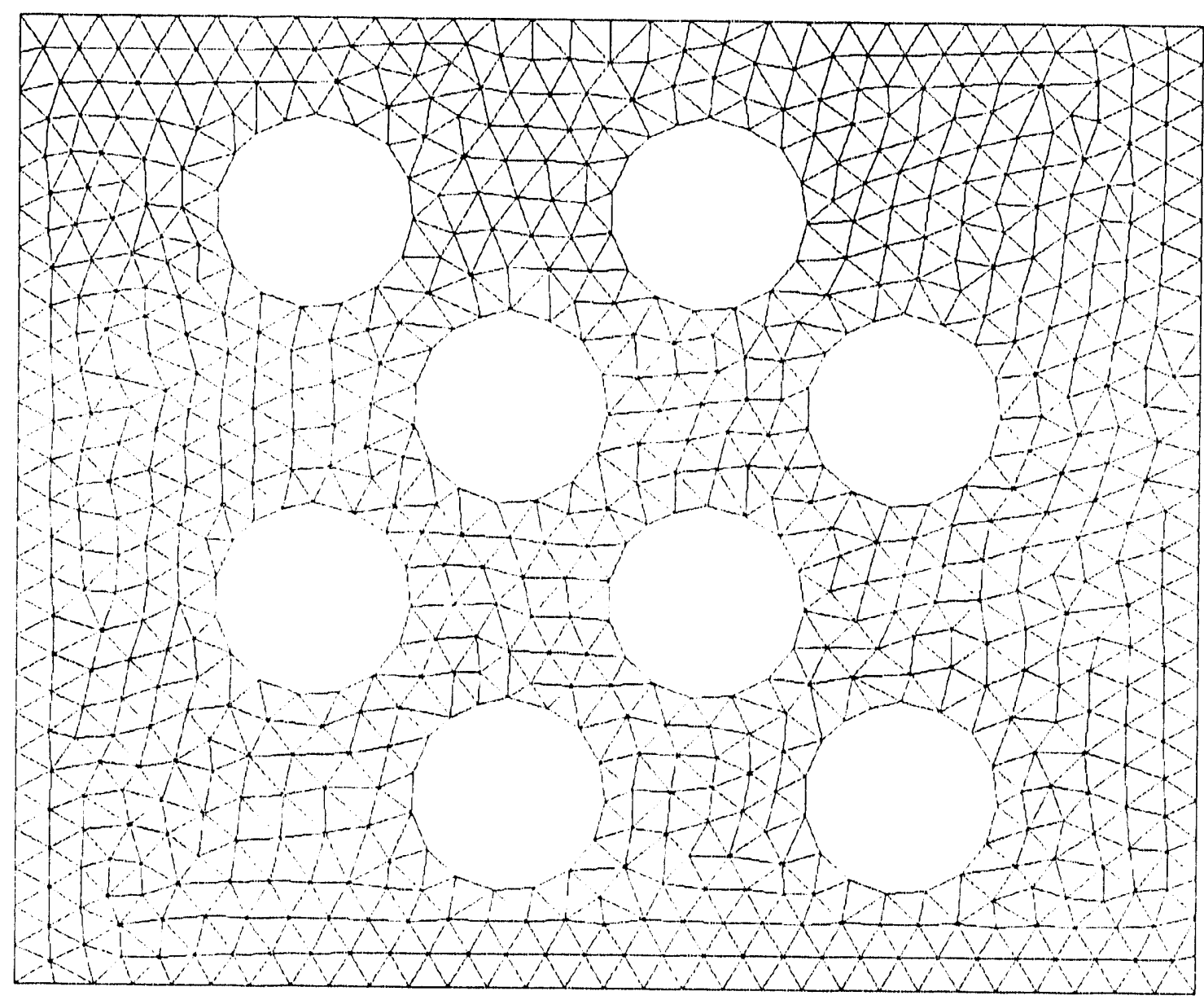

\subsubsection{String descriptor}

The string descriptor defines a string by specifying a start point coordinate values followed

1. This figure is an unstructured triangular grid of a staggered tube array. The grid was generated using GEN2D, a program written by Rainald Lohner, Navel Research Laboratory, Washington D.C., and grider, a program using the NCAR Graphics Library written by Vincent A. Mousseau, Idaho National Engineering Laboratory, Idaho Falls, ID.

My thanks go to Michael Stewart, Idaho National Engineering Laboratory, Idaho Falls, ID, for this contribution. 
by one or more connecting coordinate values. After the starting point is specified, the following coordinates can be thought of as "pen down" positions. A string descriptor begins with ' $s$ ' and is followed by two floating point values. Note the following example:

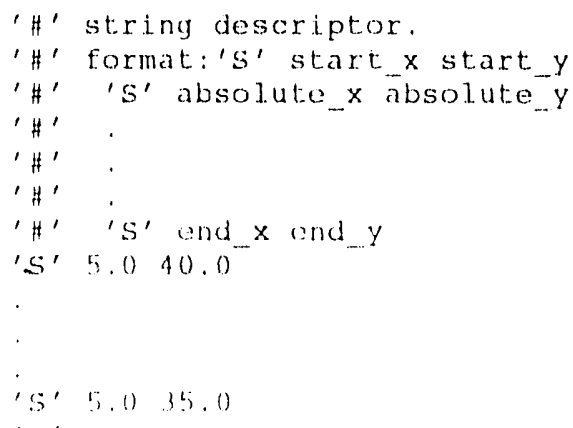

The string is terminated (pen up mode) by specifying a descriptor other than the string descriptor, like the comment, for example. The continent outline in Figure 28 was generated by using the string descriptor.

FIGURE 28. Geometry drawn using the string descriptor

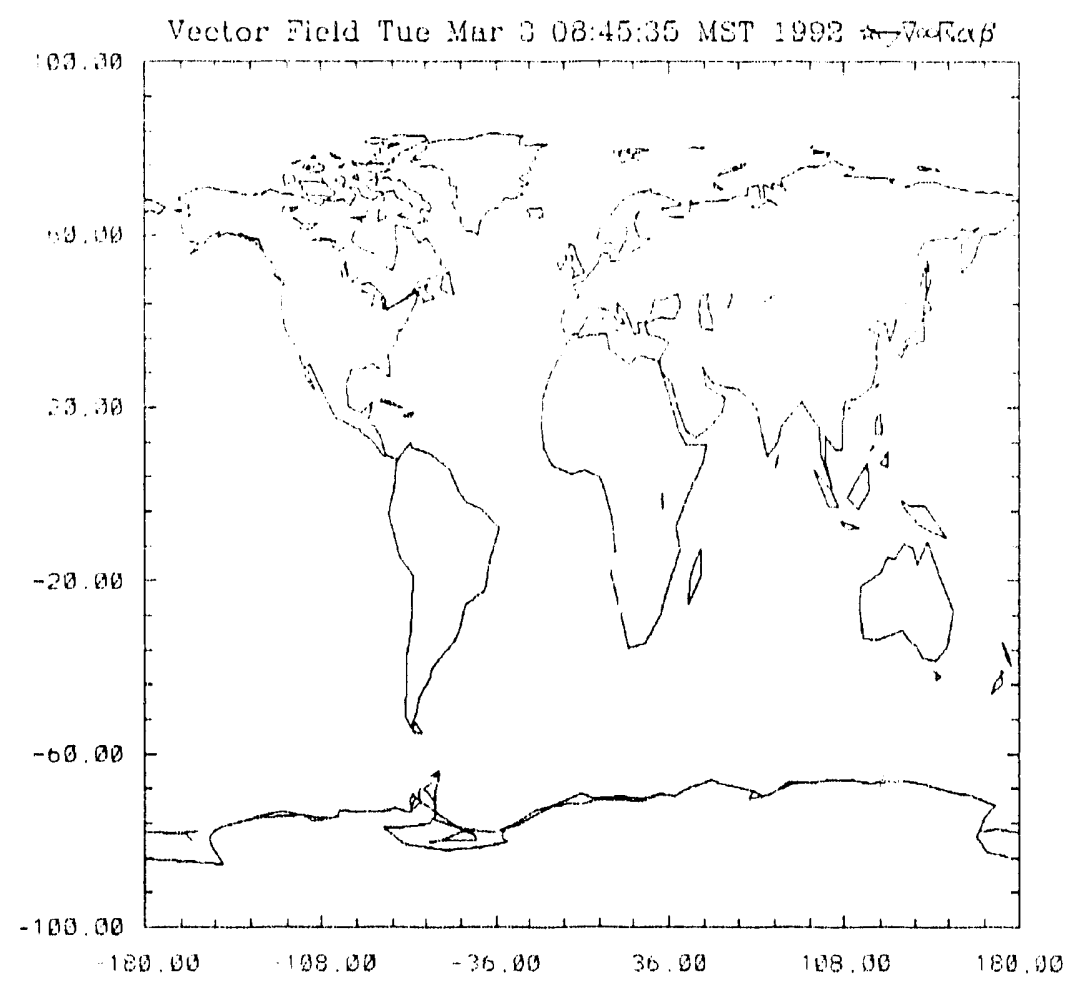




\subsubsection{Arc (circle) descriptor}

The arc descriptor (or circle descriptor) defines an arc by specifying the center point coordinate values, the arc radius and the start and end angle (in degrees). An arc descriptor begins with 'A' and is followed by five floating point values. Note the following example:

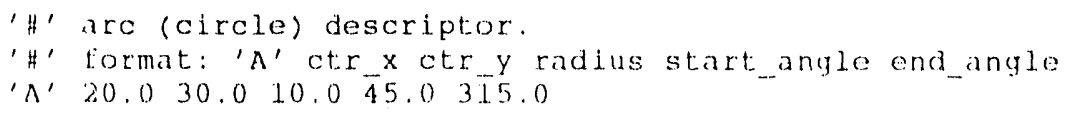

Figure 29 was generated by using the arc descriptor with a vector field.

FIGURE 29. (Geometry drawn using the arc (circle) descriptor ${ }^{2}$

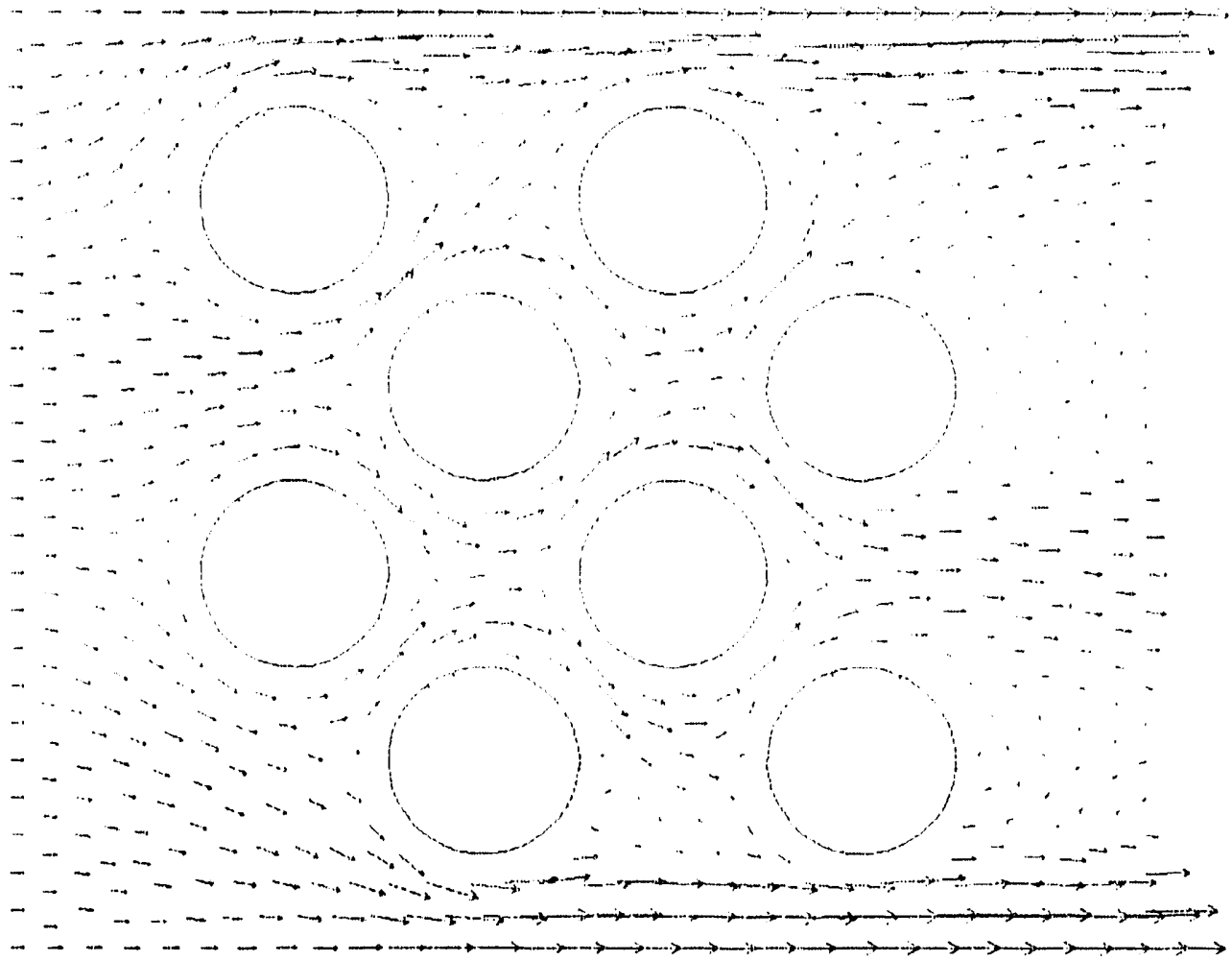

2. This figure is a 2D simulation of a flow through a staggered tube array. Velocity vectors are shown indicating direction and magnitude of the flow field. This figure shows that the effects of flow blockage around a tube bundle, decreases the efficiency of a heat exchanger. Finite Volume Compressible Flow Solver software was used to generate the vector field.

My thanks go to Michael Stewart, Idaho National Engineering Laboratory, Idaho Falls, ID, for this contribution. 


\subsubsection{Curve (spline) descriptor}

The curve descriptor (or spline descriptor) defines a spline by specifying the starting point coordinate values, followed by one or more connecting coordinate values. A curve descrip$t o r$ begins with ' $\mathrm{C}$ ' and is followed by two floating point values. Note the following example:

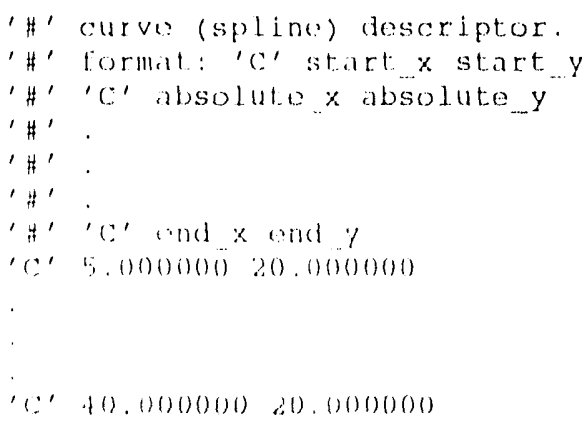

Figure 30) was generated by using the curve descriptor.

FIGURE 30. Geometry drawn using the curve (spline) descriptor

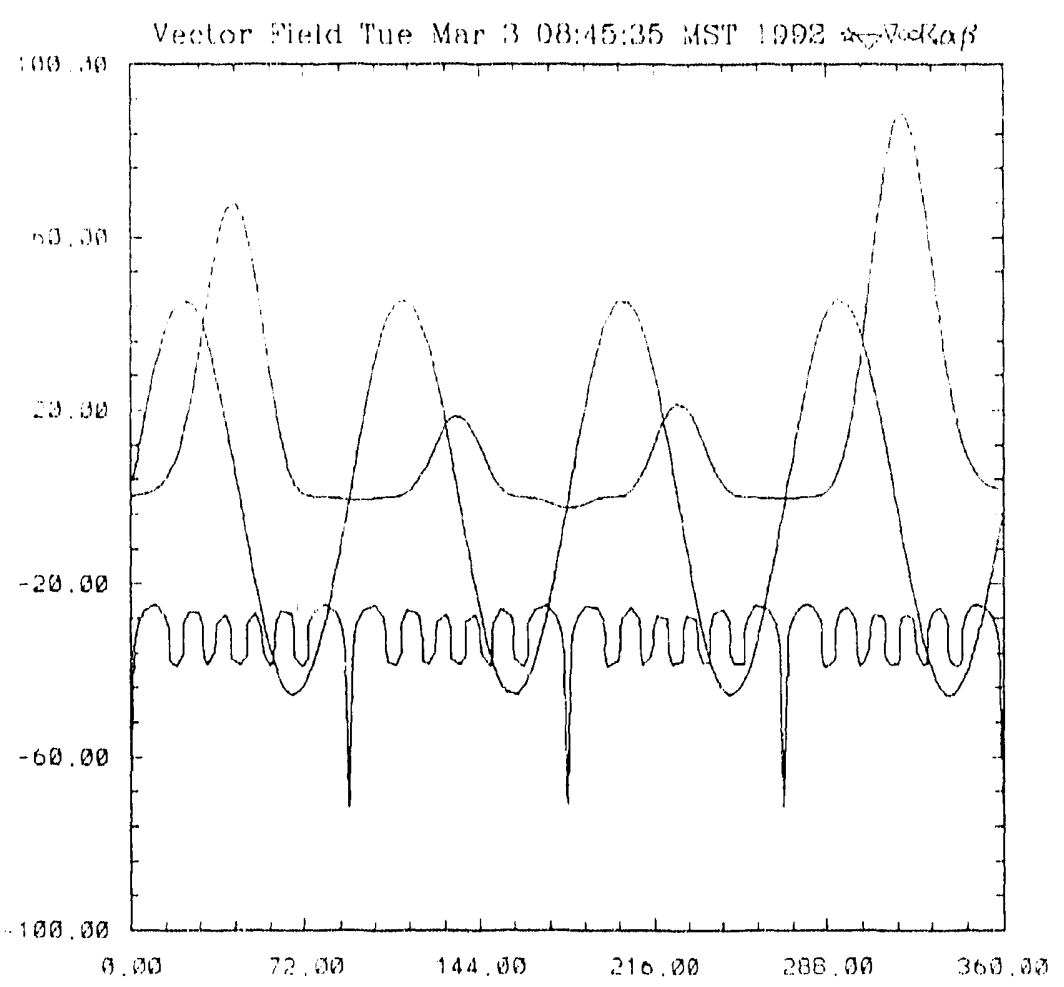




\subsection{Vector database}

The vector database gives the user the capability to define a regular, irregular, or random gridded vector field in an ASCII database. The filename is specified by the filein parameter (see Section 2.1.6, on page 6) in the parameter file.

Vectors are defined by specifying the $x / y$ coordinate values and the $u / v$ velocity component respectively. The format of the vector database is simply four floating point values on each line. The following is an example listing of a vector database:

(atmast eat voreter a dat

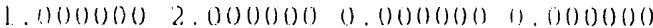

2.000000 $3.001900009 .8480 / 83 \quad 1.73648 \%$

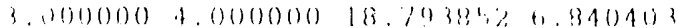

Figure 31 illustrates the individual parts of at velocity vector:

FIGURE 31. Coordinates of a velocity vector

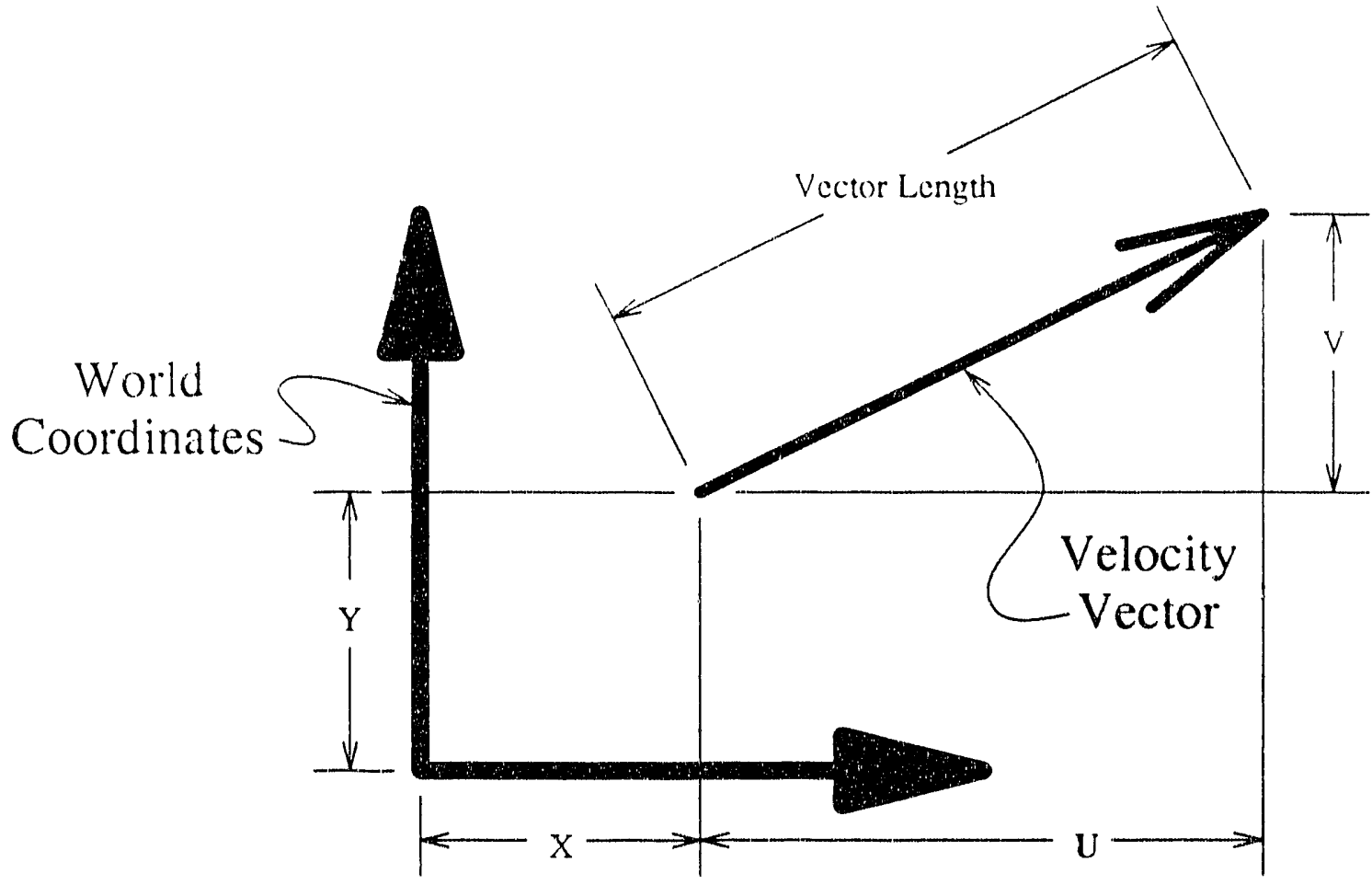




\section{Vectum Output}

The output from vectum can be displayed on a monitor (computer screen), submitted to a default or networked printer, and/or can be used to generate several different file formats. The following is a brief discussion regarding the use of the NCAR utilities to manipulate the coutput.

\subsection{Displaying to a monitor}

Ctrans is a NCAR CGM translator. It is can be used to display a NCAR CGM file to a terminal.

\subsubsection{Y11 driver}

The command syntax for displaying a metafile $t(0$ a 7()()$\times 7()()$ pixel, $X 11$ window, positioned at the upper-left comer of the screen, is as follows:

$$
\text { host? ctrans - } \mathrm{d} \times 11 \text {-geom } 700 \times 700+10+10 \text { gmeta \& }
$$

Setting the GRAPHCAP environment variable can eliminate the need for the "-d $x 11$ " device type switch on the ctrans command line. The FONTCAP can also be set with an environment variable. Note the following commands:

$$
\begin{array}{ll}
\text { host: } & \text { setenv GRAPHCAP XII } \\
\text { host? } & \text { setenv FONTCAP font } 1
\end{array}
$$

These commands can also be placed in the " $/$, cshrc" or $" \sim /, \log$ in" file and will be executed automatically upon login.

An entry can be placed in the applications defaults file to eliminate the need for the "-geom $700 \times 700+10+10 "$ geometry window specifier switch on the ctrans command line. The following entry should be placed in the " / . Xdef aults" file:

$$
\text { ctrans*geometry: } 700 \times 700+10+10
$$

The X11 driver on ctrans also allows you to change the foreground and background colors. Note the following:

$$
\begin{gathered}
\text { host? ctrans - d X11 -background pink । } \\
\text {-foreground green gmeta \&. }
\end{gathered}
$$

A list of color names and their red, green, blue values are in found in the 
Vectum User's (inide

"/usr/lib/X11/rgb.txt" lile.

\subsubsection{Tektronix driver}

Ctrans can also be used to display a NCAR metafile to a Tektronix 4115 terminal (or emulator). The command syntax is as follows:

hostz ctrans - $\mathrm{d}$ t4115 gmeta \&

The GRAPHCAP environment variable for a Tektronix 4115 terminal would be as follows:

host: setenv GRAPHCAP t4115

\subsection{Submission to a printer}

Besides displaying a NCAR CGM tile, ctrans can be used to spool the file directly to at printer: Several output device drivers are available.

\subsubsection{PostScript driver}

The syntax for spooling to a monochrome Postscript printer is as follows:

$$
\text { hosto ctrans - d ps.mono gmeta / lpr - Pprinter }
$$

The syntax for translating a CGM file to PostScript format and spooling it directly to the Imagen printer from the cray or jade is as follows:

$$
\begin{aligned}
\text { jader ctrans - a ps.mono gmeta | ipr -Pimagen } & \\
& \text { - Lultrascript }
\end{aligned}
$$

If you get real creative, (and have the proper authorizations in the " " / . rhosts" file) you can even spool a PostScript file to the Imagen from your own workstation. The following is an alias from a " / c cshrc" file:

$$
\begin{gathered}
\text { alias pstoimagen'ctrans - d ps.mono gmeta | rsh jade । } \\
\text { /usr/local/bin/ipx -Pimagen - Lultrascript' }
\end{gathered}
$$

\subsubsection{Impress driver}

The syntax for translating a CGM file to Impress format and spooling it directly to the Imagen printer from the cray or jade is as follows: 
jader ctrans - d imagen gmeta / ipr-Pimagen-Limpress

Again, with some Unix intuition, (and the proper authorizations in the " / rhosts" file) you can spool an Impress file to the Imagen from your own workstation. The following is an allias from a " / cshrc" file:

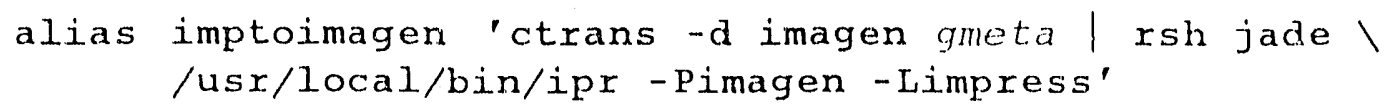

\subsubsection{HPL.J driver}

Ftrans is also a VCAR CGM translator. NCAR currently recommends that you use the utilities ctrans, ictrans, or idt in every calse except when plotting to HP Lilserdet printers. The syntix for spooling to a HP Latserjet printer is als follows:

$$
\text { host: ftrans - a hplj300p gmeta / 1pr - Pprinter }
$$

\subsection{Generating a file}

Ctrans can also be used to translate the C(BM tile into several tile tormalts. The file can then be transterred across the network to another workstation or be imported into a desktop publishing parkage llike this one ${ }^{3}$ ).

\subsubsection{PostScript file}

Crams can be used to translate the CGM tile into a Postscript file. The syntax for creating a monochrome PostScript tile is ats follows:

hostz ctrans - d ps.mono gmeta>gmeta.ps

\subsubsection{XWD file}

Ctrans can be used to translate the CGM file into X Window Dump (XWD) format. The syntax for creating a XWD file is as follows:

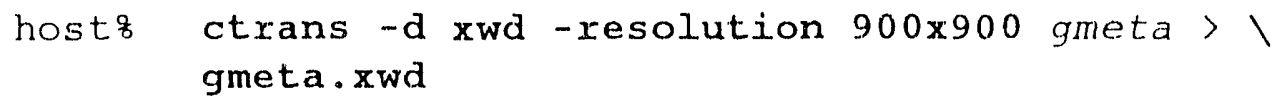

3. This documentation was generated using FrameMaker(i) Version $3.1 \mathrm{X}$ by Frame Technology Corp. 
Note that the resolution of the image can be specified by using the "-resolution" command line switch.

\subsubsection{Sun Raster file}

Ctrans can be used to translate the CGM file into Sun Raster format. The syntax for creating a Sun Ralster file is as follows:

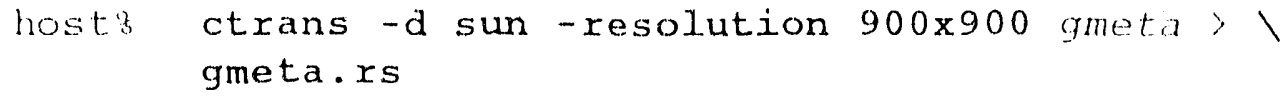

Note that the resolution of the image can be specified by using the "-resolution" command line switch.

Before importing raster output from vectum into a desk top publishing packinge, you maly wish to force the image to monochrome and then invert black lor white. This will force the background to white and the foreground (i.e. vectors, axes, labels) to black. This can be done using Jef Poskanzer's portable pixrlap (ppm), portable graymap (pglom), and portable bitmap (pbm) uilities a available by anon FTP on jalde ats

"pub/sco/utils / pbinplus tar. :"). Note the following:

host?

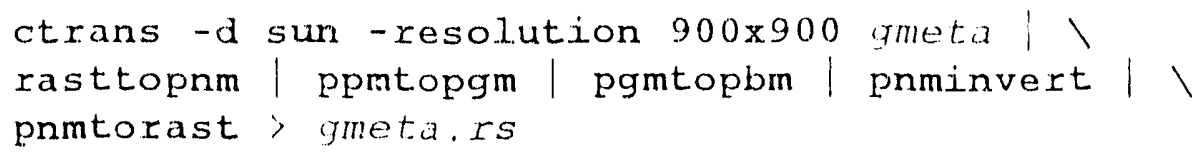

The Figure 32 was generated by using the plomplus utilities with a Sun Raster file and imported into this document. See Figure 32, on page 46 for a comparison with an imported Enciapsulated PostScript lile.

FIGURE 32. Imported Sun Rasister tile

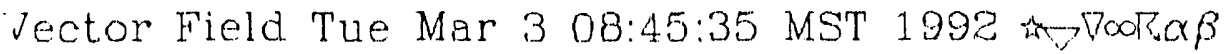

12.00 ГT, T, T, T, T, T, T, T, T, T, T,

\subsubsection{Encapsulated PostScript file}

NCAR does not have an Encapsulated PostScript driver for ctrans, however, PostScript can be converted into Encapsulated PostScript without great loss of resolution. First, generate a PostScript file:

$$
\text { hostg ctrans - d ps.mono gmeta> gmeta.ps }
$$


Then, convert the PostScript tile to Encapsulated PostSoript by using the utility pstoepsi

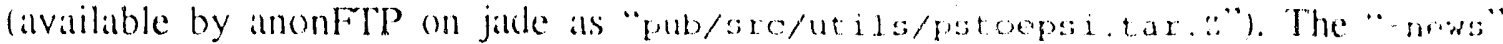
switch on pstoepsi uses Sun's $\mathcal{X} / N_{e}$ WS (OpenWindows) PostScript interpreter to perform the conversion:

$$
\text { hostz pstoepsi -news - strip gmeta.ps qmota.ens }
$$

If $X / N e W S$ is not available, the PostScript file can be rasterized using ghostseript iavailable

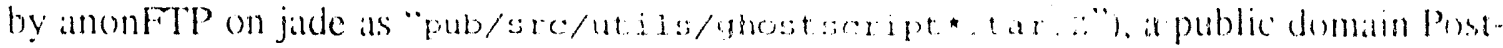
Script interpreter. The "gs" switch on pstoepsi uses shostseript on perform the conver. sicon.

$$
\text { host? pstoepsi -gs -strip meri.lis mot al ws }
$$

The figure 33 wats generated using the ". new" switch on the pstoepsi command and imported into this document. See Figure 32 , un patge 46 for a comparison wath in imported raster tile.

FICURE 33. Imported lincapsulated Pontscript tile

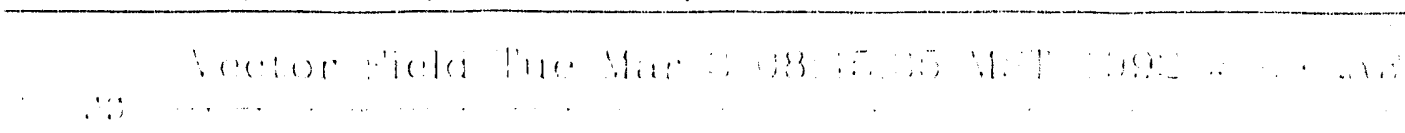


Vectum Uiser's Giuide 


\section{References}

Adobe Systems, Inc.., 1987, Encapsulated PostScript File Format Version 1.2, Mountain View, California

ANSU/ISO, 1990, "Computer Graphics Metafile", ANSI/ISO, American National Standards Institute/International Standards Organization, ISO 8632 PT 1, 2, 3, and 4-90) (Revision of ANSI X3.122-1986).

Clare, F., Kennison, D., and Lackman, B., 1987, NCAR Graphics User's Guide, Version 2.(k), Boulder, Colorado: National Center For Atmospheric Research, Scientific Computing Division.

Clare, F. and Kennison. D., 1989, NCAR Graphics Guide to New Utilities, Version 3.(0), Boulder, Colorado: National Center For Atmospheric Research, Scientific Computing Division.

Frame Technology Corporation. 1990, FrameMaker Reference. Sin Jose, California

Frame Technology Corporation, 1990. Using FrameMaker, San Jose. Callifornia

Massachusetts Institute Technology X Consortium, 1986, "X Window Dump File Format", XWDFile.h. Cambridge, Masssachusetts

Poskanzer, J., 1991,.prim,ppm,pgm,pbrn”,UNIX MamPages, jef@well.st.cit.us

Sobell, M., 1989, A Practical Guide to the UNIX System, 2nd ed., Redwood City, California: Benjamin/Cummings Publishing Company, Inc.

Sun Microsystems, Inc., 1987, "rasterfile", UNIX Man Pages, Mountain View, California

Taft, E., and Walden, J., Adobe Systems, Inc., 1990, PostScript Language Reference Manual, 2nd ed., Reading, Massachusetts: Addsion-Wesley Publishing Company. 
Vectum User's Guide 
Vectum User's Guide

\section{Glossary}

alias: A mechanism in the $C$ and Korn Shells that permits the definition of new commands. ASCII: Acronym that stands for the American National Standard Code for Information Interchange. It uses seven bits to represent letters, numbers, punctuation and control characters. ASCII is one of the most common human-readable, file formats and is used for program source code and when exchanging information between computers.

cat: Unix command to concatenate or display files.

cd: Unix command to change working directory.

CGM: Acronym that stands for Computer Graphics Metafile. CGM is a standard file formalt defined by ANSI X3.122-1986.

cshrc: A command file in the home directory that the $C$ Shell execute: each time a new shell is invoked. This file is used for establishing variables and aliases.

ctrans: A NCAR Graphics utility for translating a NCAR CGM file. It can be used to displaty the CGM file to a terminal, spool the tile directly to a printer, or convert the file into one of several file formats.

default.vec: An ASCII database in the present working directory that is read by the program vectum and affects how the program is executed. The file contains parameter information that controls details of the displayed vector field.

Encapsulated PostScript: A variation of the PostScript file format that includes a bound. ing box.

777: Unix command to access the FORTRAN 77 compiler.

Finite Volume Compressible Flow Solver: A program that solves finite volume compressible flow problems.

FrameMaker: A commercial, What-I-See-Is-What-I-Get (WISIWIG) desktop publishing packange by Frame Technology Corp., Siln Jose, CA. FrameMaker generates publication-quality documentation through at motif-like, point-ind-click user interface.

ftp: Acronym that stands for File Transfer Protocol. FTP is used to transfer files from one machine on the network to another.

ftrans: A NCAR Graphics utility for translating a NCAR CGM file to a HP LaserJet printer.

GEN2D: A program for generating an unstructured triangular grid mesh, written by Rainald Lohner, Navel Research Laboratory, Washington D.C.

ghostscript: A public domain PostScript previewer (and interpreter).

gmeta: The default CGM output filename generated by vectum and several other NCAR Graphics utilities.

grider: A program for generating an unstructured triangular grid mesh, based on the NCAR Graphics Library and written by Vincent A. Mousseau, Idaho National Engineering Laboratory, Idaho Falls, ID. 
ictrans: A command-driven, interactive NCAR Graphics utility for translating a NCAR CGM file.

idt: An X-based, point-and-click, interactive NCAR Graphics utility for translating a NCAR CGM file.

INEL: Acronym that stands for Idaho National Engineering Laboratory.

ipr: Unix command to (queue file(s) to an Imagen printer.

login: A command file in the home directory that the $C$ Shell executes each time the user logs in or gains access to a Unix system by responding correctly to the login and password prompt. This file is used to set environment variables and run commands that are executed at the beginning of each login session.

lpr: Unix command to queue file(s) to a network printer.

Is: Unix command to display intormation about one or more files.

make: Unix utility to update files. The make utility is most often used to compile and link a set of program source files.

makefile: An ASCII database, used by the make utility, that contains Unix commands and defines file relationships.

mkdir: Unix command to make a directory.

NCAR: Acronym that stands for the National Center for Atmospheric Research, an orgatnization located in Boulder, Colorado.

pbomplus: $A$ set of public domain utilities (filters) written by Jef Poskanzer for converting and modifying ratster images.

PostScript: The popular file format written by Adobe Systems, Inc., Mountain View, CA. pstoepsi: A public domain script for converting an arbitrary PostScript image to an Encapsulated PostScript image with a bitmap, suitable for incorporation into FrameMaker, LaTeX, trotf, etc.

pwd: Unix command to display pathname of the current working dirctory.

rgh.txt: The ASCII database that assigns names to red, green, blue, vallues. Those names can then be used by many X-based programs.

.rhosts: The ASCII database in the home directory for specifying remote hosts and users that are considered trusted. This file is used by several Unix programs (likf rcp, rsh, and rlogin) to bypass the standard password-based user authentication mect anism.

rm: Unix command to remove a file(s).

rsh: Unix command to execute a shell (or command) remotely on another machine.

setenv: Unix command setting an environment variable.

Sun Raster: Sun's file format for raster images.

tar: Unix command for storing or retrieving files from an archive file or tape.

vectum: An easy to use, flexible, multi-purpose, $2 \mathrm{D}$ velocity vector plotting package based on the NCAR Graphics libraries, written by Fred R. McClurg and Vincent A. Mousseau, Idaho National Engineering Laboratory, Idaho Falls, ID.

.Xdefaults: The ASCII database that allows a user to customize an X application without recompiling. 
XWD: Acronym that stands for X Window Dump. XWD is a popular raster file format among user's of the X 11 windowing environment.

zcat: Unix command that reads a compressed file and generates uncompressed output while leaving the original file intact. Zcat eliminates the need to uncompress (and later compress) a file while extracting files from a compressed tarfile, for example. 
Vectum User':s Guids: 


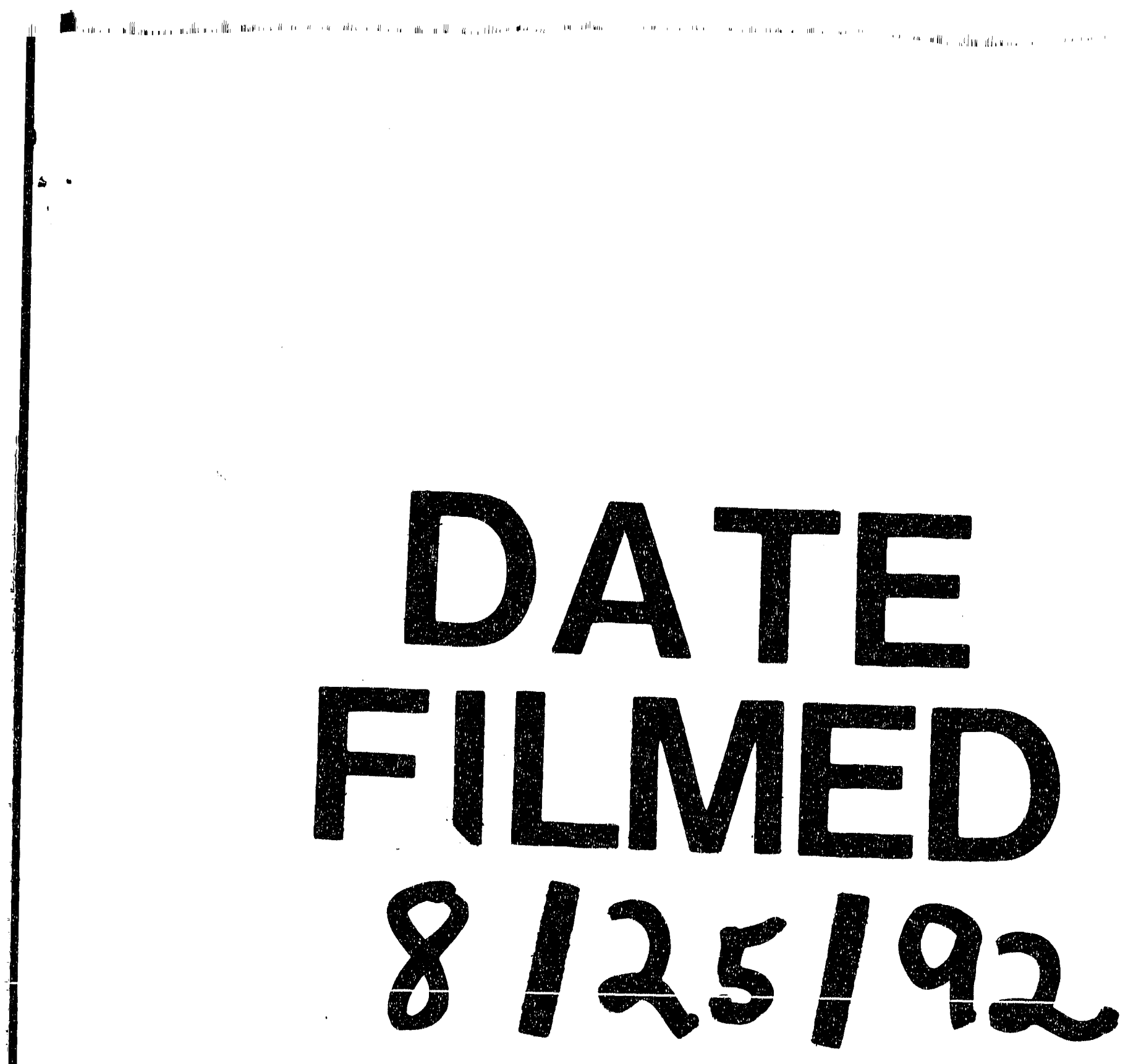


$=$ 